\title{
THREE NEW ECLIPSING WHITE-DWARF-M-DWARF BINARIES DISCOVERED IN A SEARCH FOR TRANSITING PLANETS AROUND M-DWARFS
}

\author{
Nicholas M. Law ${ }^{1,11}$, Adam L. Kraus ${ }^{2,12}{\text {, Rachel } \text { Street }^{3} \text {, Benjamin J. Fulton }}^{3}$, Lynne A. Hillenbrand ${ }^{4}$, \\ Avi ShPorer ${ }^{3,5}$, Tim Lister ${ }^{3}$, Christoph Baranec ${ }^{4}$, Joshua S. Bloom ${ }^{6}, \mathrm{Khanh} \mathrm{Bui}^{4}$, Mahesh P. Burse $^{7}$, \\ S. Bradley Cenko ${ }^{6}$, H. K. Das ${ }^{7}$, Jack. T. C. Davis ${ }^{4}$, Richard G. Dekany ${ }^{4}$, Alexei V. FilipPenko ${ }^{6}$, Mansi M. Kasliwal ${ }^{8}$, \\ S. R. Kulkarni ${ }^{4}$, Peter Nugent ${ }^{9}$, Eran O. OfeK ${ }^{4}$, Dovi Poznanski ${ }^{10}$, Robert M. Quimby ${ }^{4}$, A. N. Ramaprakash ${ }^{7}$, \\ ReEd Riddle $^{4}$, JefFrey M. Silverman ${ }^{6}$, Suresh Sivanandam ${ }^{1,11}$, and Shriharsh P. TendulKar ${ }^{4}$ \\ ${ }^{1}$ Dunlap Institute for Astronomy and Astrophysics, University of Toronto, 50 St. George St., Toronto, ON M5S 3H4, Canada \\ 2 Institute for Astronomy, University of Hawaii, 2680 Woodlawn Drive, Honolulu, HI 96822, USA \\ ${ }^{3}$ Las Cumbres Observatory Global Telescope Network, Inc., 6740 Cortona Dr. Suite 102, Santa Barbara, CA 93117, USA \\ ${ }^{4}$ Cahill Center for Astrophysics, California Institute of Technology, Pasadena, CA 91125, USA \\ ${ }^{5}$ Department of Physics, Broida Hall, University of California, Santa Barbara, CA 93106, USA \\ ${ }^{6}$ Department of Astronomy, University of California, Berkeley, CA 94720-3411, USA \\ ${ }^{7}$ Inter-University Centre for Astronomy and Astrophysics, Ganeshkhind, Pune-411007, India \\ ${ }^{8}$ Carnegie Institution for Science, 813 Santa Barbara St., Pasadena, CA 91101, USA \\ ${ }^{9}$ Computational Cosmology Center, Lawrence Berkeley National Laboratory, 1 Cyclotron Road, Berkeley, CA 94720, USA \\ ${ }^{10}$ School of Physics and Astronomy, Tel-Aviv University, Tel Aviv 69978, Israel \\ Received 2011 December 2; accepted 2012 July 30; published 2012 September 11
}

\begin{abstract}
We present three new eclipsing white-dwarf/M-dwarf binary systems discovered during a search for transiting planets around M-dwarfs. Unlike most known eclipsing systems of this type, the optical and infrared emission is dominated by the M-dwarf components, and the systems have optical colors and discovery light curves consistent with being Jupiter-radius transiting planets around early M-dwarfs. We detail the PTF/M-dwarf transiting planet survey, part of the Palomar Transient Factory (PTF). We present a graphics processing unit (GPU)-based boxleast-squares search for transits that runs approximately $8 \times$ faster than similar algorithms implemented on general purpose systems. For the discovered systems, we decompose low-resolution spectra of the systems into white-dwarf and M-dwarf components, and use radial velocity measurements and cooling models to estimate masses and radii for the white dwarfs. The systems are compact, with periods between 0.35 and 0.45 days and semimajor axes of approximately $2 R_{\odot}(0.01 \mathrm{AU})$. The M-dwarfs have masses of approximately $0.35 M_{\odot}$, and the white dwarfs have hydrogen-rich atmospheres with temperatures of around $8000 \mathrm{~K}$ and have masses of approximately $0.5 M_{\odot}$. We use the Robo-AO laser guide star adaptive optics system to tentatively identify one of the objects as a triple system. We also use high-cadence photometry to put an upper limit on the white-dwarf radius of $0.025 R_{\odot}$ ( $95 \%$ confidence) in one of the systems. Accounting for our detection efficiency and geometric factors, we estimate that $0.08 \%{ }_{-0.05 \%}^{+0.10 \%}$ (90\% confidence) of M-dwarfs are in these short-period, post-common-envelope white-dwarf/M-dwarf binaries where the optical light is dominated by the M-dwarf. The lack of detections at shorter periods, despite near-100\% detection efficiency for such systems, suggests that binaries including these relatively low-temperature white dwarfs are preferentially found at relatively large orbital radii. Similar eclipsing binary systems can have arbitrarily small eclipse depths in red bands and generate plausible small-planet-transit light curves. As such, these systems are a source of false positives for M-dwarf transiting planet searches. We present several ways to rapidly distinguish these binaries from transiting planet systems.
\end{abstract}

Key words: binaries: eclipsing - methods: data analysis - planets and satellites: detection - stars: low-mass techniques: photometric - white dwarfs

Online-only material: color figures

\section{INTRODUCTION}

Large numbers of non-eclipsing white-dwarf/main-sequence binaries have been discovered in the Sloan Digital Sky Survey (SDSS) and other surveys (e.g., Rebassa-Mansergas et al. 2012; Bianchi et al. 2007, and references therein). For low-mass stars in particular there is a bridge in color between white dwarfs and M-dwarfs. The bridge is interpreted as being due to rare white-dwarf/M-dwarf binaries, at a ratio with respect to single stars of $\sim 1: 2300$ (Smolčić et al. 2004).

White-dwarf/M-dwarf eclipsing systems are much rarer, and almost all have been discovered by searching for white dwarfs

\footnotetext{
${ }^{11}$ Dunlap Fellow.

${ }^{12}$ Hubble Fellow.
}

displaying very deep eclipses of up to several magnitudes (e.g., Nelson \& Young 1970; Green et al. 1978; Fulbright et al. 1993; Bruch \& Diaz 1998; Drake et al. 2010). These searches find systems containing relatively hot $(>12,000 \mathrm{~K})$ white dwarfs and mid-to-late M-dwarfs. The discovery rate of these systems (e.g., Drake et al. 2010; Parsons et al. 2011, 2012) is increasing with the advent of large sky surveys. These binaries survived the common-envelope phase of their evolution and many will become cataclysmic variables (e.g., Nebot Gómez-Morán et al. 2011), and so the properties and number statistics of these systems can provide windows into two important areas of stellar evolution. Precision measurements of the systems allow the determination of the masses and radii of two types of stars for which there are relatively few measurements (Nebot GómezMorán et al. 2009; Kraus et al. 2011; Pyrzas et al. 2012, 
2009), along with a verification of close binary evolution models (Pyrzas et al. 2009).

In this paper, we present three eclipsing white-dwarf/ M-dwarf systems discovered during the PTF/M-dwarfs search for transiting planets around M-dwarfs. In contrast to most known eclipsing systems of this type, the systems detected in this survey have optical and infrared emission dominated by the M-dwarf component and contain relatively low-temperature $(8000 \mathrm{~K})$ white dwarfs and relatively early M-dwarfs. The shape of the light curves of the detected systems is similar to that expected for transiting giant planets around M-dwarfs, in particular in having a flat-bottomed eclipse with a depth of 1\%-20\% in red optical bands.

The PTF/M-dwarfs survey (Law et al. 2011) is a search for transiting planets around 100,000 M-dwarfs. The survey is performed with the Palomar Transient Factory (PTF) camera (Rahmer et al. 2008; Law et al. 2009a, 2010a) on the 48 inch Samuel Oschin telescope at Palomar Observatory and is a Key Project of the PTF (Law et al. 2009a; Rau et al. 2009). The PTF/M-dwarfs survey is designed to complement other M-dwarf transiting planet surveys such as MEarth (e.g., Charbonneau et al. 2009; Irwin et al. 2010), the WFCam transit survey (Sipöcz et al. 2011), and the M-dwarfs in the Kepler mission target list (Borucki et al. 2011), by covering a much larger number of M-dwarfs at somewhat lower sensitivity. The survey achieves photometric precisions of a few percent for $\sim 100,000$ targets, and few-millimag precision around a subset of $\sim 10,000 \mathrm{M}$-dwarfs. These systems offer much larger transit depths compared to solar-type stars, while their very red colors compared to most other stars in the field greatly reduce the probability of a blended eclipse producing a difficult-to-detect transit false positive.

The three eclipsing systems presented here were originally detected as Jupiter-sized exoplanet candidates during the first year of operations of the survey. Followup of the candidates showed large color changes during eclipse and very large radial velocity (RV) signals, suggesting a hidden hot companion. In this paper, we detail the properties of these eclipsing whitedwarf/M-dwarf systems and explore ways to distinguish them from true planetary transits.

The paper is organized as follows. In Section 2, we describe the PTF/M-dwarfs survey, its precision photometry methods, and its target detection strategies, including a new method of performing a box-least-squares (BLS) transit search in parallel on Graphics Processing Unit (GPU) hardware. Section 3 details the three new eclipsing white-dwarf/M-dwarf systems and describes follow-up photometric, low-resolution spectroscopic, and RV observations, which are used to determine masses and radii for the system components in Section 4. Section 5 determines the frequency of eclipsing binaries such as these and discusses ways to distinguish them from transiting exoplanets.

\section{THE PTF/M-DWARFS SURVEY}

The PTF/M-dwarfs project consists of a transiting planet survey on the robotic 48 inch Samuel Oschin telescope (hereafter P48), photometric follow-up using the Palomar 60 inch telescope, the Byrne Observatory at Sedgwick Reserve (hereafter BOS), the LCOGT Faulkes-North and Faulkes-South telescopes (FTN and FTS), and RV follow-up with the High-Resolution Echelle Spectrometer (HIRES; Vogt et al. 1994) instrument on Keck I.

The $8 \mathrm{deg}^{2}$ camera (with 7.26 useful square degrees) on the $\mathrm{P} 48$ telescope allows the survey to cover $\approx 3000 \mathrm{M}$-dwarfs in
Table 1

The Specifications of the PTF Camera and the PTF/M-dwarfs Survey

\begin{tabular}{|c|c|}
\hline \multicolumn{2}{|c|}{ P48 PTF camera specifications } \\
\hline Telescope & Palomar 48 inch (1.2 m) Samuel Oschin \\
\hline Camera field dimensions & $3.50 \times 2.31$ \\
\hline Camera field of view & $8.07 \mathrm{deg}^{2}$ \\
\hline Light-sensitive area & $7.26 \mathrm{deg}^{2}$ \\
\hline Image quality & 2.0 arcsec FWHM in median seeing \\
\hline Filters & $g^{\prime}$ and Mould- $R$; other bands available \\
\hline CCD specs & $2 \mathrm{~K} \times 4 \mathrm{~K}$ MIT/LL 3 edge-butted CCDs \\
\hline Plate scale & $1^{\prime \prime} .01$ pixel $^{-1}$ \\
\hline Readout noise & $<12 \mathrm{e}^{-}$ \\
\hline Readout speed & $35 \mathrm{~s}$, entire $100 \mathrm{MPix}$ array \\
\hline \multicolumn{2}{|c|}{ PTF/M-dwarfs survey characteristics } \\
\hline Targets & Late-K, M, and L dwarfs with $m_{R}<18$ \\
\hline Survey sky area & $29 \mathrm{deg}^{2}$ every 2 months \\
\hline Target locations & $20^{\circ}<$ Galactic latitude $<35^{\circ}$ \\
\hline Targets covered & $\approx 12,000$ every 2 months \\
\hline Observations per night & $5 \mathrm{hr}$ \\
\hline Exposure time & $60 \mathrm{~s}$ \\
\hline Cadence & 15-25 minutes \\
\hline Observation length & $1-3$ months \\
\hline Efficiency & $66 \%$ open shutter (slew during readout) \\
\hline Saturation level & $m_{R} \approx 14$ (seeing dependant, in $\left.60 \mathrm{~s}\right)$ \\
\hline \multirow[t]{2}{*}{ Sensitivity (median) } & $m_{R} \approx 21$ in $60 \mathrm{~s}, 5 \sigma$ \\
\hline & $m_{g^{\prime}} \approx 21.3$ in $60 \mathrm{~s}, 5 \sigma$ \\
\hline \multirow[t]{2}{*}{ Photometric stability } & 3 mmag (brightest targets) \\
\hline & $10 \%$ (faintest targets) \\
\hline \multirow[t]{3}{*}{ Followup } & Photometric: Palomar 60 inch, FTN/S, BOS \\
\hline & Low-res spectroscopic: Lick Shane-3 m \\
\hline & Radial velocity: Keck I/HIRES \\
\hline
\end{tabular}

each pointing (Figure 1), for a total of around 100,000 targets per year at Galactic latitudes of $20^{\circ}-35^{\circ}$. The PTF/M-dwarfs survey typically observes several fields with an approximately 20 minute cadence for $4-5 \mathrm{hr}$ per night. Individual fields are typically observed for several months, and observations are performed throughout the year. All PTF/M-dwarfs data are acquired with a 60 s exposure time in the PTF camera's Mould- $R$ filter (similar to the SDSS $r$ filter). Data taking is interleaved with the PTF supernova survey which generally operates on 1-3 days cadences, and so scheduling constraints lead to a variety of final cadences for PTF/M-dwarfs fields. Table 1 summarizes the specifications of the PTF camera and the PTF/M-dwarfs survey.

The PTF/M-dwarfs survey is sensitive to equal-mass binaries around all M-dwarfs in the survey fields brighter than $m_{R} \approx 20$. In practice, the mass and radius determination and follow-up observation of the faintest systems is extremely challenging, so we impose a discovery magnitude limit of $m_{R} \approx 18$. At that magnitude, the typical $\mathrm{P} 48$ data photometric precision is a few percent per data point, allowing immediate high-precision constraints on the system properties in discovery data. Given the saturation and faint cutoff limits of the survey, its effective distance ranges are 200-1300 pc for M0 dwarfs, 50-290 pc for M5 dwarfs, and 10-70 pc for M9 dwarfs.

\subsection{P48 Data Reduction}

Reduction of the PTF/M-dwarfs data is performed in two steps: the standard PTF real-time data reduction software first calibrates the images, and then a custom pipeline performs source extraction, association, and precision photometry. 


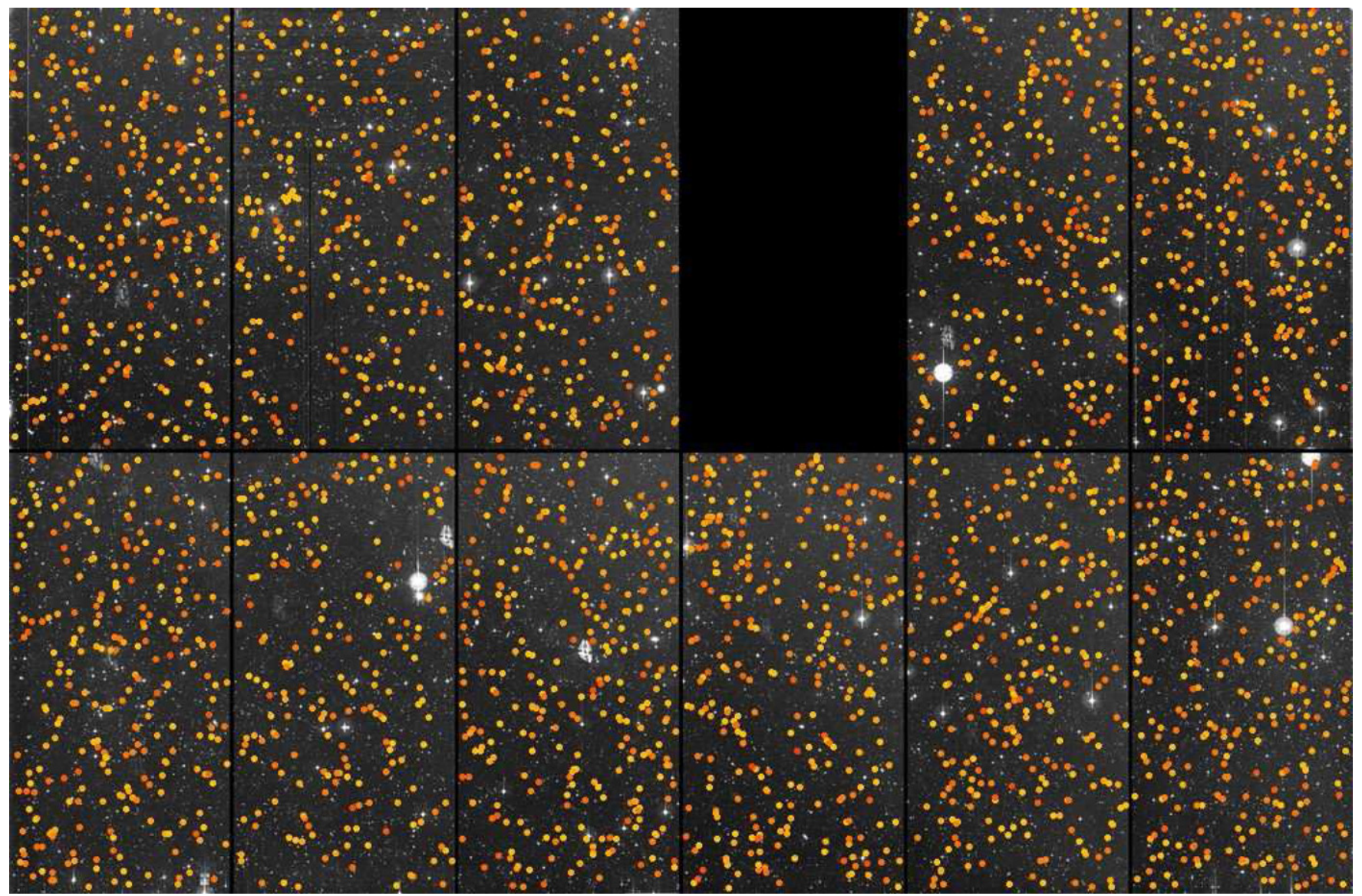

Figure 1. PTF camera image of a survey field centered at $\alpha=17: 28, \delta=+57: 22$, and covering $3.50 \times 2.31$. The highlighted points show the 2851 stars with photometrically estimated spectral types later than K4 and photometric stability better than $5 \%$. The colors of the points correspond to the stellar temperatures, with $\mathrm{K} 4$ as yellow and late-M-dwarfs as red. The pipeline has removed stars with possible photometric precision problems such as proximity to a bright star or a bright ghost image. North is up and east is to the right.

(A color version of this figure is available in the online journal.)

Immediately after observations, PTF data are transferred to Lawrence Berkeley National Laboratory where cross-talk corrections are applied to each chip, standard bias/overscan subtraction is performed, and a superflat based on recently acquired data is applied.

After the calibrated data are transferred to the Dunlap Institute for Astronomy and Astrophysics at University of Toronto, the PTF/M-dwarfs pipeline extracts sources from the calibrated images, produces an optimal photometric solution, associates sources in images taken at different times, and applies a range of eclipse-detection algorithms to the resulting light curves. We summarize the system here; the pipeline is described in more detail in Law et al. (2011).

Initial source extraction is performed on individual CCD chip images by SExtractor (Bertin \& Arnouts 1996) using radius-optimized aperture photometry with a locally optimized background. The extracted sources are filtered to remove those close to bad pixels, diffraction spikes from bright stars, and those that may be affected by nearby sources. Heliocentric Julian dates (HJD) are used for time measurements throughout the pipeline.

The photometric zero points for each epoch are initially estimated based on either SDSS or USNO-B1 photometry for bright stars in the field. The pipeline then optimizes the zero point of each epoch to minimize the median photometric variability of all the remaining sources. This first optimization typically improves the long-term photometric stability to below the percent level. The pipeline then filters the generated light curves, search- ing for epochs that produce anomalous photometry for a large fraction of the sources; those epochs are usually those affected by clouds, moonlight, or some other effect that varies across the images. Typically $0 \%-2 \%$ of epochs are flagged by this process and are removed from further consideration. A second iteration of variable-source removal and zero-point optimization is then performed. The final zero points are applied to each light curve, along with flags for poor conditions, nearby sources that could cause confusion, bad pixels, and other problems that could affect the photometry. Running on a $2.5 \mathrm{GHz}$ quad-core desktop computer the pipeline processes a 300 epoch set of 11 chips (54 GB of image data) in less than $24 \mathrm{hr}$.

The pipeline typically achieves a photometric stability of 3-5 mmag over periods of months (Figure 2). The photometric precision is photon limited for all sources fainter than $m_{R} \approx 16$, except in regions of crowding or nebulosity. The pipeline has been used for several PTF programs such as the open cluster rotation project (Agüeros et al. 2011).

\subsection{Eclipsing Binary Detection}

The pipeline produces light curves for $25,000-100,000$ stars per field. The large number of light curves makes the processing time involved in searching for eclipses an important consideration. An initial cut is made on the basis of the estimated spectral type of the source, based on photometry from the USNO-B1, Two Micron All Sky Survey (2MASS), and (where 


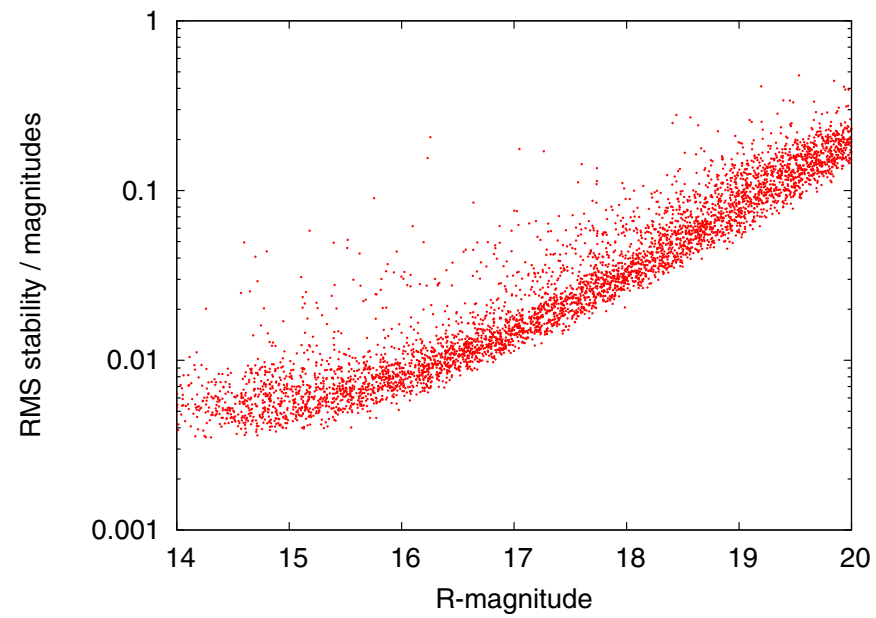

Figure 2. Photometric stability achieved in a typical PTF/M-dwarfs field in 140 epochs spanning 92 days of observations. Each point corresponds to the root-mean-square (rms) variability of one of the 23,713 stars in this field with magnitude $14<m_{R}<20$. For clarity, $50 \%$ of the fainter stars have been removed from this plot. The best stability achieved in this field is $4 \mathrm{mmag}$, with some CCD chips and sky regions having somewhat lower stability at 5-7 mmag. The few stars with very high variability compared to others at similar magnitudes are astrophysically varying sources such as eclipsing binaries, RR Lyrae stars, and other variables.

(A color version of this figure is available in the online journal.)

available) SDSS data (Monet et al. 2003; Skrutskie et al. 2006; York et al. 2000). The photometric data are fit to updated versions of the spectral energy distributions (SEDs) given in Kraus \& Hillenbrand (2007), yielding an accuracy of approximately 1 subclass. Sources with late-K, M, or L estimated spectral types are passed to our eclipse-search algorithms.

\subsubsection{High-variability Source Searches}

Many eclipsing binaries have eclipse signals in the tens-ofpercent range as well as large eclipse duty cycles. These systems have significantly increased photometric variability compared to nearby stars of similar magnitude, and so a simple variability search can rapidly find them (Figure 2). We estimate the value and scatter of the locally expected photometric stability as a function of stellar magnitude in 0.1 mag bins, using a sigmaclipped average of the stars detected on each chip. Objects that show a more than $2 \sigma$ increased variability compared to the ensemble expectation are flagged for further review. Of the systems presented here, only PTFEB11.441 was detected in this manner; the photometric variations from the smaller eclipse depths of the other systems required more computationally intensive algorithms.

\subsubsection{A Parallel Eclipse Search Using Graphics Processing Units}

Systems with smaller eclipse depths and/or longer periods and reduced duty cycles may have only slightly increased photometric variability, necessitating a more sensitive search. We use a standard BLS (Kovács et al. 2002; Tingley 2003) algorithm to phase the light curves at all possible periods and search for a transit-like signal.

This algorithm requires the testing of thousands of periods and transit phases and is thus computationally expensive. However, the problem is easily parallelizable as an arbitrary number of light curves and periods can be tested simultaneously. We take advantage of this by implementing the BLS algorithm on a GPU that can perform hundreds of computations in parallel.
Our BLS search is run on an NVIDIA Tesla C2050 GPU that contains 448 cores operating at $1.15 \mathrm{GHz}$, for a total of 1.03 Tflops in single-precision floating point arithmetic. The BLS search is coded in CUDA and is called from the PyCUDA python module (Klöckner et al. 2009).

Perhaps the simplest parallel-processing technique for the BLS algorithm is to have each GPU thread perform all the calculations for one source; in this way several hundred sources could be tested simultaneously. However, the BLS algorithm requires at least one access to the full light-curve data at each test period. The per-thread memory in typical GPUs is too small to contain a full light curve, necessitating frequent calls to the GPU global memory. These calls are slow, even when synchronized across threads, greatly limiting the speed of the GPU implementation.

Instead, we consider sources sequentially, and simultaneously test hundreds of different periods on a single source. The processing of each light curve proceeds as follows. First, the light-curve data are read into fast shared memory by each thread block (with memory accesses coalesced for speed). Next, each thread picks an untested period, phases the light curve at that period and then tests a range of durations and phases.

In testing with typical PTF/M-dwarfs data, our GPU BLS algorithm operates approximately eight times faster than a multithreaded program executing on all cores of an Intel Core-2 Quad Core CPU running at $2.50 \mathrm{GHz}$. With the GPU approximately 10,000 light curves can be fully searched for transit or eclipse events each hour.

\section{DISCOVERIES AND FOLLOW-UP OBSERVATIONS}

We discovered three eclipsing M-dwarf/white dwarf binary systems in a search of $\approx 45,000 \mathrm{M}$-dwarfs from the first year of PTF/M-dwarf operations. Each system was detected as a high-confidence planet candidate, with a 5\%-20\% depth flatbottomed eclipse in the $R$ band with duration consistent with a transiting Jupiter-radius planet. Images of the field around each target are shown in Figure 3, and the P48 detection light curves of the systems are shown in Figure 4.

The PTF names of the three detected sources are PTF1 J004546.0+415030.0, PTF1 J015256.6+384413.4, and PTF1 J015524.7+373153.8. For brevity, we hereafter refer to the sources by their PTF/M-dwarfs survey internal names, which are based on their decimal right-ascension coordinates: PTFEB11.441, PTFEB28.235, and PTFEB28.852, respectively.

\subsection{Follow-up Measurements}

After the systems were detected in PTF data as planet candidates we followed the standard PTF/M-dwarfs followup strategy: high-cadence multi-color photometry with the Palomar 60 inch, BOS, and FTN, along with RV observations with the HIRES spectrograph on Keck I. The multi-color photometry (Section 3.1.1) rapidly revealed that these objects had a strongly varying eclipse depth with color (Figures 5 and 6), suggesting we were seeing eclipses between two self-luminous objects with different temperatures. Low-resolution spectra (Section 3.1.4) were sufficient to immediately confirm a whitedwarf component in one system (PTFEB11.441), but required detailed modeling to recover the white-dwarf components in the other two systems (Section 4.1). RV observations were scheduled to allow determinations of the white-dwarf mass. 


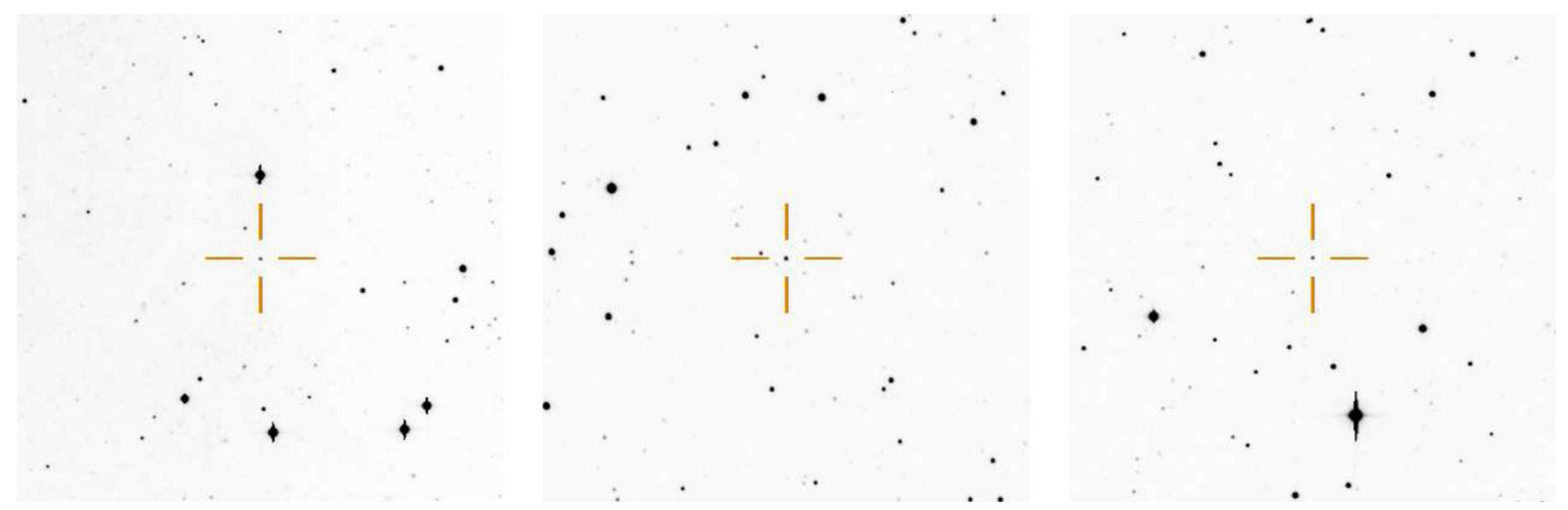

Figure 3. Images of the newly discovered systems taken with the PTF camera (left-to-right: PTFEB11.441, PTFEB28.235, and PTFEB28.852). North is up and east is to the left; each image shows a $400 \times 400$ arcsec cutout from the $3.50 \times 2.31$ PTF camera field.

(A color version of this figure is available in the online journal.)
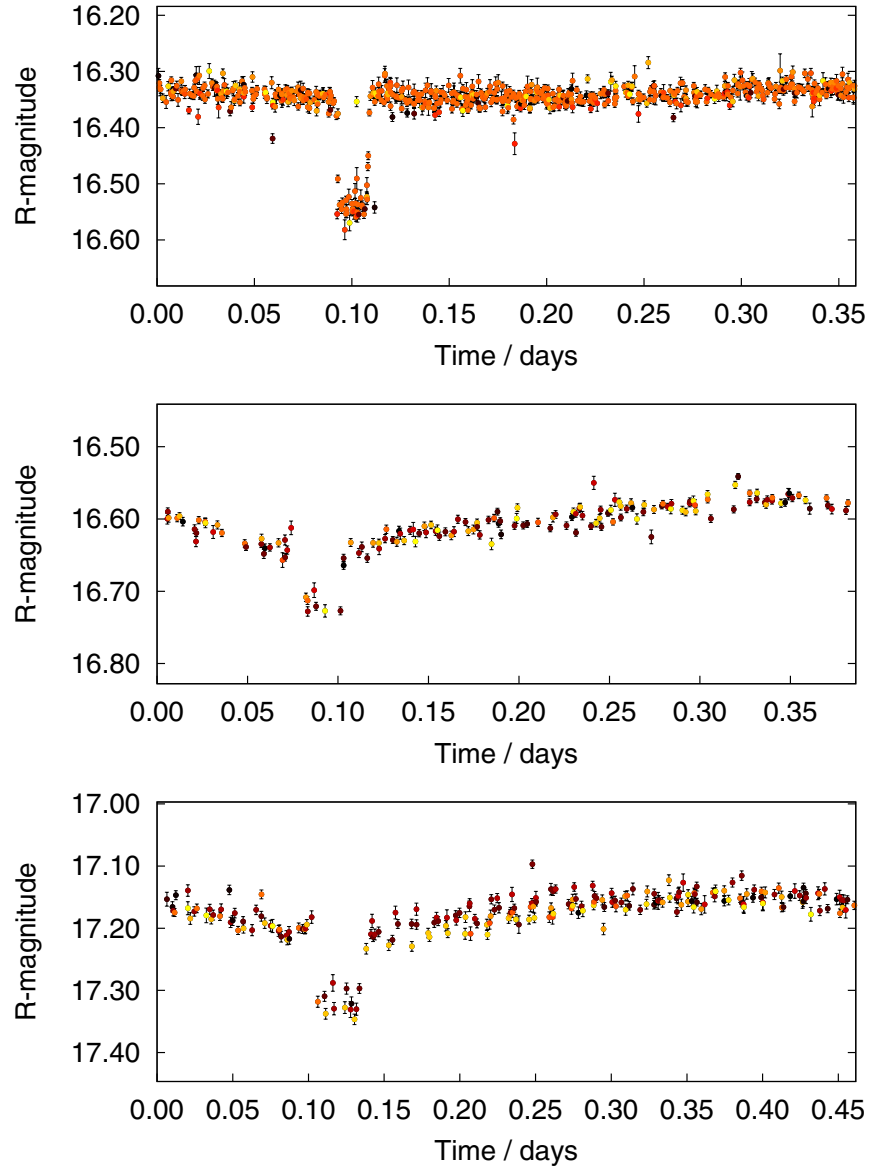

Figure 4. Discovery P48 light curves for the three eclipsing systems (top-tobottom: PTFEB11.441, PTFEB28.235, and PTFEB28.852). The colors of the points correspond to the time the data point was measured: black are the oldest points and bright yellow are the newest. PTFEB11.441 was observed during a high cadence run targeted at M31, hence the large density of points all taken on a single night.

(A color version of this figure is available in the online journal.)

\subsubsection{High-cadence, Multi-color Photometry with BOS}

Multi-color photometry data for each of the targets were gathered with the RC Optics $0.8 \mathrm{~m}$ telescope at Byrne Observatory at Sedgwick reserve near Santa Ynez, CA. The BOS telescope is equipped with a Santa Barbara Instrument Group STL-6303E

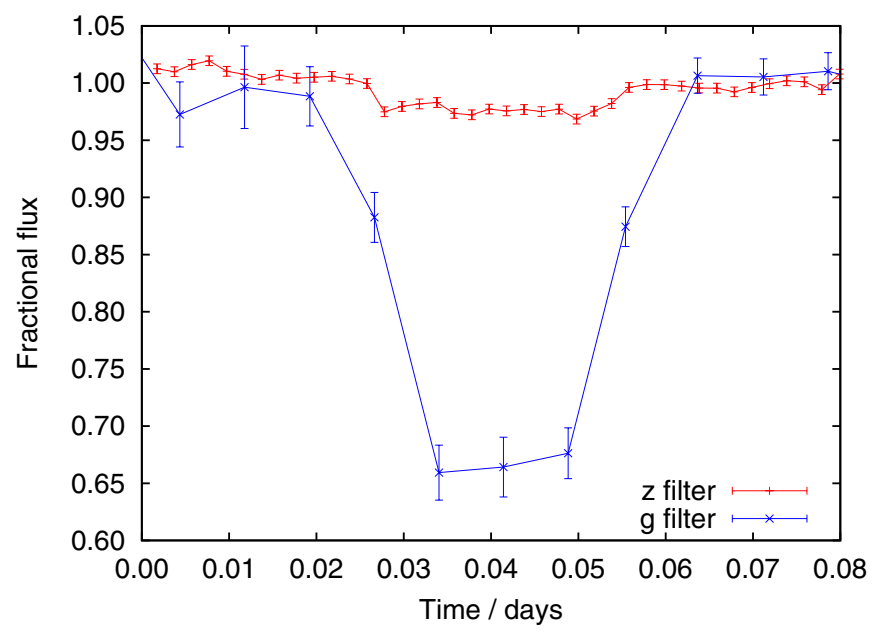

Figure 5. Faulkes-North ( $z$ filter) and BOS ( $g$ filter) follow-up photometry of PTFEB28.852. The eclipse is detected at high significance in both bands, but is approximately $10 \times$ deeper in the $g$ filter, suggesting the secondary eclipse of a hot body.

(A color version of this figure is available in the online journal.)

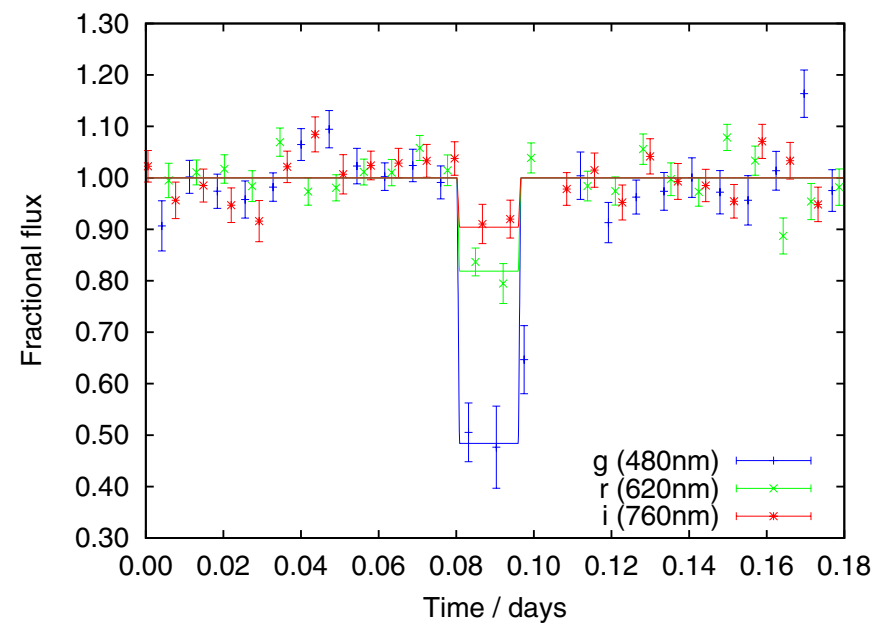

Figure 6. Dunlap Institute Arctic Telescope photometry of PTFEB11.441, showing the varying eclipse depth with wavelength. The $g, r$, and $i$ filters (with midpoints shown in the figure) were alternated in sequence to produce a nearly simultaneous multi-color light curve. To guide the eye, each color has a fit to the light curve using the system's eclipse parameters derived in Section 4.5.

(A color version of this figure is available in the online journal.) 
camera utilizing a $3 \mathrm{k} \times 2 \mathrm{k}$ Kodak Enhanced KAF-6306E CCD, with a $14.7 \times 9$ 9.8 field of view and a pixel scale of $0^{\prime} .572 \mathrm{pixel}^{-1}$ $(2 \times 2$ binning). We observed individual eclipses in a single color with SDSS $i^{\prime}$, SDSS $g^{\prime}$, or Astrodon Photometrics UV-blocked clear filters.

The images were reduced using standard routines for bias subtraction, dark-current subtraction, and flat-field correction. We extracted fluxes for all stars in the frame using aperture photometry routines in PyRAF. Relative-flux light curves were produced by dividing the flux of the target star by the summed flux from several comparison stars in each image. Julian dates of mid-exposure were recorded during the observations, and later converted to barycentric Julian dates using the online tools described by Eastman et al. (2010). Aperture sizes were optimized by minimizing the scatter of the resulting light curves and ranged from $3^{\prime \prime}$ to $6^{\prime \prime}$. Exposure times were typically $300 \mathrm{~s}$.

Several eclipses of PTF11.441 were observed in shortcadence mode at BOS. We used an Astrodon Photometrics UV-blocked clear filter in order to maximize the signal-to-noise ratio $(\mathrm{S} / \mathrm{N})$ per unit time. We used $3 \times 3$ binning $\left(0^{\prime \prime} .858\right.$ pixel $\left.^{-1}\right)$, and set the camera to only readout a small subsection of the CCD encompassing the target star, and three nearby stars of similar brightness to be used as comparison stars. This reduced the readout plus dead time of the instrument from $\approx 10 \mathrm{~s}$ to $\approx 5 \mathrm{~s}$, and allowed us to achieve a photometric noise rate ${ }^{13}$ of $\approx 1.6 \% \mathrm{~min}^{-1}$.

\subsubsection{Faulkes-North Photometry}

PTF28.852 was observed photometrically on 2010 November 10 and 16 using the $2.0 \mathrm{~m}$ FTN (Maui, Hawai'i) operated by LCOGT. In both cases, the Spectral CCD imager ${ }^{14}$ was used along with a Pan-STARRS- $z$ filter. The Spectral instrument contains a Fairchild CCD 486 back illuminated $4096 \times 4096$ pixel CCD which was binned $2 \times 2$ giving 0'.303 pixels and a field of view of $10^{\prime} \times 10^{\prime}$. Exposure times were 130 and $150 \mathrm{~s}$, respectively, on the two nights.

The frames were pre-processed using standard techniques for bias subtraction and flat-fielding; dark-current subtraction was not performed as it is negligibly small for this instrument. Object detection and aperture photometry were performed using the DAOPHOT photometry package within the IRAF environment. The adopted aperture sizes were 7 and 5 pixels in radius on the two nights. Differential photometry was performed relative to 5-6 comparison stars within the field of view.

\subsubsection{Dunlap Institute Arctic Telescope Photometry}

The Dunlap Institute Arctic Telescope is a 20 inch robotic telescope currently undergoing testing at the New Mexico Skies observatory at Cloudcroft, NM. Once testing is complete, the telescope will be based at the PEARL research station on Ellesmere Island at a latitude of $80^{\circ}$ North, where it will perform a search for transiting planets. The telescope is equipped with a 16 megapixel Apogee U16M camera with a $34^{\prime} \times 34^{\prime}$ field of view.

We observed PTFEB11.441 on the night of 2011 October 23. 120-s exposures in the $g, r$, and $i$ band were taken in sequence

\footnotetext{
13 Photometric noise rate, calculated as $\mathrm{rms} / \sqrt{(}(\Gamma)$, where rms is the scatter of the out-of-eclipse section of the light curve and $\Gamma$ is the median number of cycles (exposure time and dead time) per minute. See Shporer et al. (2010b) for a more detailed description.

$14 \mathrm{http}: / / \mathrm{www}$. specinst.com
}

throughout an eclipse of the target. After standard calibrations, differential photometry was performed using the pipeline described in Section 2.1.

\subsubsection{Low-resolution Spectra}

We obtained low-resolution optical spectra of PTFEB28.235 and PTFEB28.852 on UT 2010 November 30, and PTFEB11.441 on UT 2011 October 25. Spectra were acquired with the dual-arm Kast spectrograph (Miller \& Stone 1993) on the $3 \mathrm{~m}$ Shane telescope at Lick Observatory. The spectra used a 2 arcsec wide slit, a 600/4310 grism on the blue side, and a 300/7500 grating on the red side, yielding full width at half-maximum (FWHM) intensity resolutions of $\approx 4 \AA$ and $\approx 10 \AA$. All observations were aligned along the parallactic angle (Filippenko 1982) to reduce differential light losses.

All spectra were reduced using standard techniques (e.g., Foley et al. 2003). Routine CCD processing and spectrum extraction were completed within IRAF, and the data were extracted with the optical algorithm of Horne (1986). We obtained the wavelength scale from low-order polynomial fits to calibration-lamp spectra. Small wavelength shifts were then applied to the data after cross-correlating a template sky to the night-sky lines that were extracted with the target stars. Using our own IDL routines, we fit spectrophotometric standard-star spectra to the data to flux calibrate our spectra and to remove telluric lines (Wade \& Horne 1988; Matheson et al. 2000).

\subsubsection{Keck/HIRES Radial-velocity Observations}

We obtained high-dispersion spectra of the white-dwarf/ M-dwarf systems using the HIRES on the Keck-I $10 \mathrm{~m}$ telescope. HIRES is a single-slit echelle spectrograph permanently mounted on the Nasmyth platform. All observations were performed using the red channel and $\mathrm{C} 2$ decker, and spanned a wavelength range of 4300-8600 A or 5000-7900 $\AA$, yielding a spectral resolution of $R \sim 45,000$. We processed our HIRES data using the standard pipeline MAKEE ${ }^{15}$ which automatically extracts, flat-fields, and wavelength-calibrates spectra taken in most standard HIRES configurations. We measured the RVs for each order of each spectrum using the broadening function deconvolution algorithm described by Rucinski (1999), fitting each spectral order with either one or two components. We calculated the broadening function with respect to a bright RV standard star of similar spectral type, and then fit the peak(s) with Gaussian functions. We computed the weighted mean RV for each epoch by weighting each order according to its dispersion (across all epochs) about these mean RVs. The weights and means were computed iteratively until they converged to static values.

In order to remove small drifts in the wavelength calibration over the course of the night, we cross-correlated each spectrum's telluric features at both 6800 and $7600 \AA$ with the telluric features for standard stars selected from Nidever et al. (2002), placing all observations into a common frame set by Earth's atmosphere. As we have previously shown (Kraus et al. 2011), this reduces the systematic uncertainty in the measured velocities to $\sim 0.3 \mathrm{~km} \mathrm{~s}^{-1}$, although the achieved precision for the systems presented here is reduced by a combination of rotational broadening for this short-period systems, spot-based RV jitter, and possible effects of irradiation by the white dwarf. The results are summarized in Table 2.

\footnotetext{
15 http://spider.ipac.caltech.edu/staff/tab/makee
} 

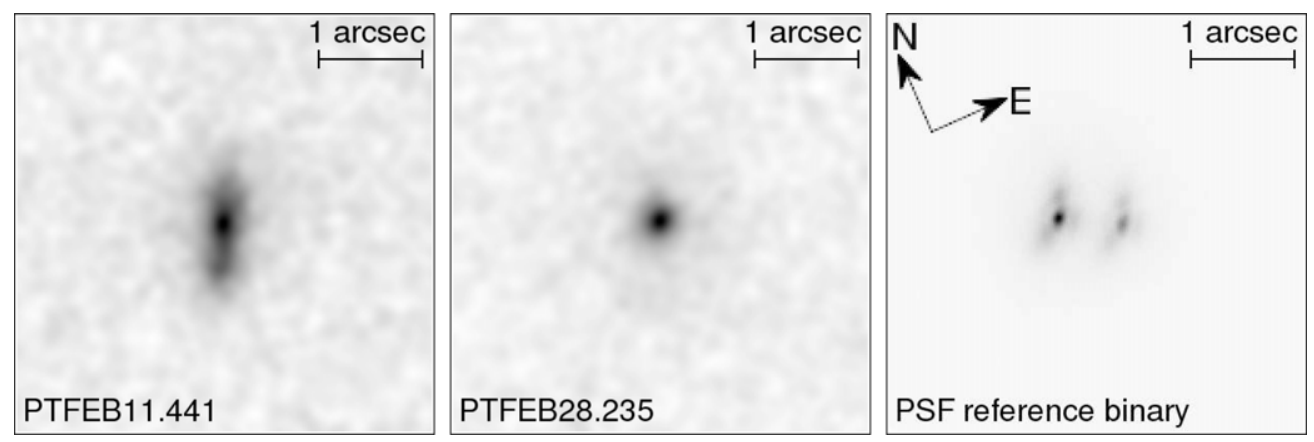

Figure 7. Robo-AO laser guide star adaptive optics images of (left-to-right) PTFEB11.441, PTFEB28.235, and an $m_{r} \approx 13$ binary taken in the same part of the night to provide a comparison point-spread function. All images are in a $600 \mathrm{~nm}$ long-pass filter, are $4^{\prime \prime}$ square, have the same rotation, and are displayed with linear scaling. To improve the S/N for these faint targets, the PTFEB11.441 and PTFEB28.235 images have been smoothed by a Gaussian filter with an FWHM corresponding to the diffraction-limited resolution of the telescope. The apparent vertical smearing of the PTFEB11.441 image is due to the shift-and-add algorithm occasionally locking on to the secondary component of the binary (Law 2007).

Table 2

High-resolution Spectroscopic Observations

\begin{tabular}{lcccr}
\hline \hline Name & $\begin{array}{c}\text { Epoch } \\
\text { (HJD) }\end{array}$ & Phase & $\begin{array}{c}t_{\text {int }} \\
(\mathrm{s})\end{array}$ & $\begin{array}{c}v_{\text {MD }} \\
\left(\mathrm{km} \mathrm{s}^{-1}\right)\end{array}$ \\
\hline PTFEB11.441 & 2455740.038314 & 0.117 & 600 & 122 \\
PTFEB11.441 & 2455740.065688 & 0.193 & 600 & 174 \\
PTFEB11.441 & 2455740.103983 & 0.300 & 600 & 172 \\
PTFEB11.441 & 2455741.037311 & 0.902 & 600 & -90 \\
PTFEB28.235 & 2455542.784023 & 0.113 & 600 & 104 \\
PTFEB28.235 & 2455542.870228 & 0.336 & 600 & 134 \\
PTFEB28.235 & 2455542.962517 & 0.575 & 600 & -86 \\
PTFEB28.235 & 2455543.756163 & 0.630 & 600 & -152 \\
PTFEB28.235 & 2455543.840477 & 0.849 & 600 & -161 \\
PTFEB28.235 & 2455741.095549 & 0.729 & 600 & -192 \\
PTFEB28.852 & 2455499.938676 & 0.287 & 900 & 111 \\
PTFEB28.852 & 2455500.056015 & 0.540 & 900 & -55 \\
PTFEB28.852 & 2455500.092409 & 0.620 & 600 & -125 \\
PTFEB28.852 & 2455500.906137 & 0.383 & 600 & 79 \\
PTFEB28.852 & 2455542.776319 & 0.104 & 600 & 71 \\
PTFEB28.852 & 2455543.943688 & 0.633 & 600 & -149 \\
\hline
\end{tabular}

\subsubsection{Robo-AO Laser Guide Star Adaptive Optics Observations}

Robo-AO is a visible and near-infrared laser guide star adaptive optics system specifically engineered for 1-3 m class telescopes (Baranec et al. 2011). The Robo-AO system comprises an ultraviolet Rayleigh laser guide star, an integrated adaptive optics and science camera system, and a robotic control system. The system currently incorporates both an electron-multiplying $\mathrm{CCD}$ and an InGaAs infrared array camera for imaging.

Robo-AO obtained images of PTFEB11.441 and PTFEB28.235 during a commissioning run at the Palomar 60 inch telescope on the night of 2011 November 18 UT. RoboAO was operated without tip-tilt correction, instead relying on post-facto shift-and-add processing of the individual frames. We used a long-pass filter with a $600 \mathrm{~nm}$ cut-on to obtain increased signal compared to a bandpass filter, along with relatively long $1 \mathrm{~s}$ exposure times to compensate for the faintness of the targets $\left(m_{R}>16.8\right)$. We used the Lucky Imaging plus adaptive optics pipeline described by Law et al. (2009b) to perform the image alignment, along with frame selection at the $10 \%$ level to boost the imaging resolution. After processing, images with approximately 0.2 FWHM point-spread functions were obtained on these targets during $\sim 1^{\prime \prime}$ seeing conditions. These observations were limited by residual tip-tilt errors during the $1 \mathrm{~s}$ exposures; similar bright targets observed the same night with $33 \mathrm{~ms}$ ex- posures had a diffraction-limited angular resolution of $\approx 0.13$ arcsec FWHM (Figure 7).

The Robo-AO imaging reveals a bright companion to PTFEB11.441, located at $0.43 \pm 0.06$ arcsec and at a position angle of $211^{\circ} \pm 3^{\circ}$ east of north.

\subsection{The Nature of the Detected Objects}

Multi-color through-eclipse photometry of the three detected systems showed that the eclipse depths were much larger in blue bands than in red bands. The sharp ingress and egress of the eclipses suggest a small body, while the flat-bottomed shape argues against that body occulting the M-dwarf, as limb darkening would then be apparent. RV observations showed a very large amplitude of more than $100 \mathrm{~km} \mathrm{~s}^{-1}$. These observations suggest the presence of a small, hot, massive object undergoing total eclipses in these systems. The white-dwarf/Mdwarf binary scenario is consistent with all these observations.

The properties of the systems are summarized in Table 3. We detail each system below.

\subsubsection{PTFEB11.441}

This system shows a possible companion in Robo-AO images. If it is associated, the companion is quite wide, at approximately 80 AU separation. There are no other sources detected in the Robo-AO image, which covers $1600 \operatorname{arcsec}^{2}$. On this basis, the probability of an unassociated star appearing within $2^{\prime \prime}$ of our target is less than $1 \%$. Definitive confirmation will require colors and common-proper-motion measurements, but for the purposes of the following sections we tentatively associate the companion star with PTFEB11.441.

The two wide components of the system have roughly equal brightness and the low-resolution spectrum of the system is well fit by a combination of a white dwarf and an M-dwarf spectrum without further components (Section 4.1), suggesting that the spectral types of the two M-dwarfs are very similar. Wide M-dwarf binaries make up a small fraction of the binary population, but many examples have been found (Dhital et al. 2010), and a very large fraction of the components of the widest binaries have close companions with roughly equal masses (Law et al. 2010b). However, we note that the M-dwarf near the white dwarf in PTFEB11.441 survived the commonenvelope phase of the system's evolution, and so it is unlikely that this system formed in the same manner as other known wide M-dwarf binaries.

PTFEB11.441 lies in a PTF field targeted at M31 and is located approximately $1^{\circ}$ from the center of the galaxy. Of 
Table 3

Measured and Estimated System Properties

\begin{tabular}{|c|c|c|c|c|c|c|c|c|c|c|}
\hline Name & $\alpha(\mathrm{J} 2000)$ & $\delta(\mathrm{J} 2000)$ & Period & $\begin{array}{c}T_{0} \\
(\mathrm{HJD})\end{array}$ & $\begin{array}{l}\mathrm{SpT}_{\mathrm{MD}} \\
( \pm 0.5)\end{array}$ & $\begin{array}{c}\text { Phot.SpT } \\
( \pm 0.5)\end{array}$ & $\begin{array}{c}T_{\mathrm{WD}} \\
( \pm 500 \mathrm{~K})\end{array}$ & Dist./pc & $M_{\mathrm{MD}}$ & $R_{\mathrm{MD}}$ \\
\hline PTFEB11.441 & 004546.0 & +415030.0 & $0.35871(5) \mathrm{d}$ & $2455438.317(5)$ & M3 & M3.5 & $8500 \mathrm{~K}$ & $180 \pm 45^{*}$ & $0.35 \pm 0.05 M_{\odot}$ & $0.33 \pm 0.05 R_{\odot}$ \\
\hline PTFEB28.235 & 015256.6 & +384413.4 & $0.3861(1) \mathrm{d}$ & $2455460.111(5)$ & M3 & M3 & $8000 \mathrm{~K}$ & $200 \pm 50$ & $0.35 \pm 0.05 M_{\odot}$ & $0.33 \pm 0.05 R_{\odot}$ \\
\hline PTFEB28.852 & 015524.7 & +373153.8 & $0.46152(9) \mathrm{d}$ & $2455530.733(5)$ & M3 & M2 & $8500 \mathrm{~K}$ & $260 \pm 70$ & $0.35 \pm 0.05 M_{\odot}$ & $0.33 \pm 0.05 R_{\odot}$ \\
\hline
\end{tabular}

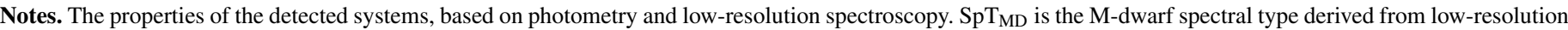

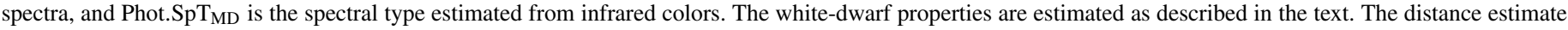

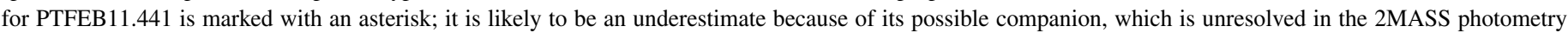
used to generate the distance estimate.

the three systems, PTFEB11.441 shows the greatest amount of emission from the white dwarf, with the M-dwarfs being overwhelmed at wavelengths $\lesssim 550 \mathrm{~nm}$. The light curve shows only very low level out-of-eclipse variability. The target is detected by GALEX with a near-UV (NUV) magnitude of $m_{\mathrm{NUV}}=17.35$, with no far-UV (FUV) detection.

\subsubsection{PTFEB28.235}

The P48 light curve of this system shows out-of-eclipse variability at the $7 \%-10 \%$ level, almost as strong as the eclipse itself in the $R$ band. The variability phasing (maximal when the white dwarf is in front of the M-dwarf) suggests that the variability is primarily an irradiation effect. The 5\% eclipse depth is the smallest $R$-band depth of the three systems. The system has a GALEX NUV detection at $m_{\mathrm{NUV}}=21.6 \pm 0.4$ (with no FUV coverage).

\subsubsection{PTFEB28.852}

This system is very similar to PTFEB28.235, with comparable variability levels and a similar light-curve shape. The system has a GALEX FUV detection at $m_{\mathrm{FUV}}=21.0 \pm 0.1$ (with no NUV coverage).

\section{MASS AND RADIUS MODELS AND MEASUREMENTS}

The optical light in these systems is dominated by the M-dwarfs, and the large radius ratios between the components produce very small (millimag-level) primary eclipses. For these reasons, we were able to measure precise eclipse shapes only for the white-dwarf occultation events, and RVs for the M-dwarf components. However, the low-resolution spectra allow us to estimate the mass and radius of the M-dwarf component of the system and thus estimate the properties of the white-dwarf component. Finally, high-cadence photometry of the ingress and egress of the white-dwarf occultation events allowed us to set stringent upper limits on the radius of the white dwarf in PTFEB11.441.

\subsection{Primary and Secondary Spectra}

We model the low-resolution spectra of our targets as a combination of a M-dwarf and a white-dwarf spectrum. Template M-dwarf spectra are taken from the HILIB stellar flux library (Pickles 1998) and have steps of a single spectral subclass. The white-dwarf models ${ }^{16}$ were kindly provided by Detlev Koester and are on a grid with $250 \mathrm{~K}$ steps between $6000 \mathrm{~K}$ and $10,000 \mathrm{~K}$

\footnotetext{
16 Koester (2010). Balmer lines in the models were calculated with the modified Stark broadening profiles of Tremblay \& Bergeron (2009), kindly made available by the authors.
}

(and larger steps up to $20,000 \mathrm{~K}$ ), and 0.25 dex steps between $\log g=6.0$ and $\log g=9.5$, where the units of $g$ are $\mathrm{cm} \mathrm{s}^{-2}$.

We simultaneously fit the white-dwarf and M-dwarf spectra using a downhill simplex algorithm and a bootstrap (e.g., Press et al. 1992) method to find the uncertainties in the fit. Figure 8 shows the fitted models and each component of the binaries with the model for the other component subtracted. In each case subtraction of the template M-dwarf spectrum revealed a (noisy) DA white-dwarf spectrum with clear Balmer lines.

PTFEB11.441 is well fit by a simple combined white-dwarf and M-dwarf spectrum, suggesting that the third component in the system is also an M-dwarf, and that the two M-dwarfs have similar spectral types. In the following sections, we use the measured spectral type for the M-dwarfs in PTFEB11.441, assuming that it is the spectral type of the M-dwarf closest to the white dwarf.

\subsection{Reddening}

The M-dwarf spectral types measured from the twocomponent fit to the system spectra closely agree with those estimated from infrared photometry of the systems (Table 3). This suggests that reddening is not a significant problem for the M-dwarf spectral types and thus for the mass and radius estimates as well.

However, we note that reddening will affect the estimated white-dwarf temperature more severely as its flux is mostly in the blue end of the spectrum. The estimated galactic extinction along the line of sight to PTFEB11.441 is $E(B-V)=0.02$ (Schlegel et al. 1998) and will be lower for the target itself which is located at a distance of $\approx 400 \mathrm{pc}$. The other two targets are located in regions of higher line-of-sight extinction, at $E(B-V)=1.5$ and $1.1 \mathrm{mag}$ for PTFEB28.235 and PTFEB28.852, respectively. The actual extinction for the two targets is, however, likely to be much lower as they are located at distances of only $\approx 200 \mathrm{pc}$. This is supported by several different arguments: the very good match of the M-dwarf model spectrum to the observed spectrum; the match between the infrared-colorestimated spectral types and the spectrally measured spectral types; and the GALEX detections of both objects, which require a small NUV and FUV extinction.

\subsection{M-dwarf Properties}

We fit the M-dwarf masses, radii, and distances in the following manner. We first fit survey photometry of the system with the stellar SEDs listed in Kraus \& Hillenbrand (2007), yielding a photometric spectral type and distance. Despite the availability of other photometric data such as USNO-B1, we restrict our fits to 2 MASS $J, H$, and $K$ colors that are unlikely to be affected by the light from the white dwarf. We assume 

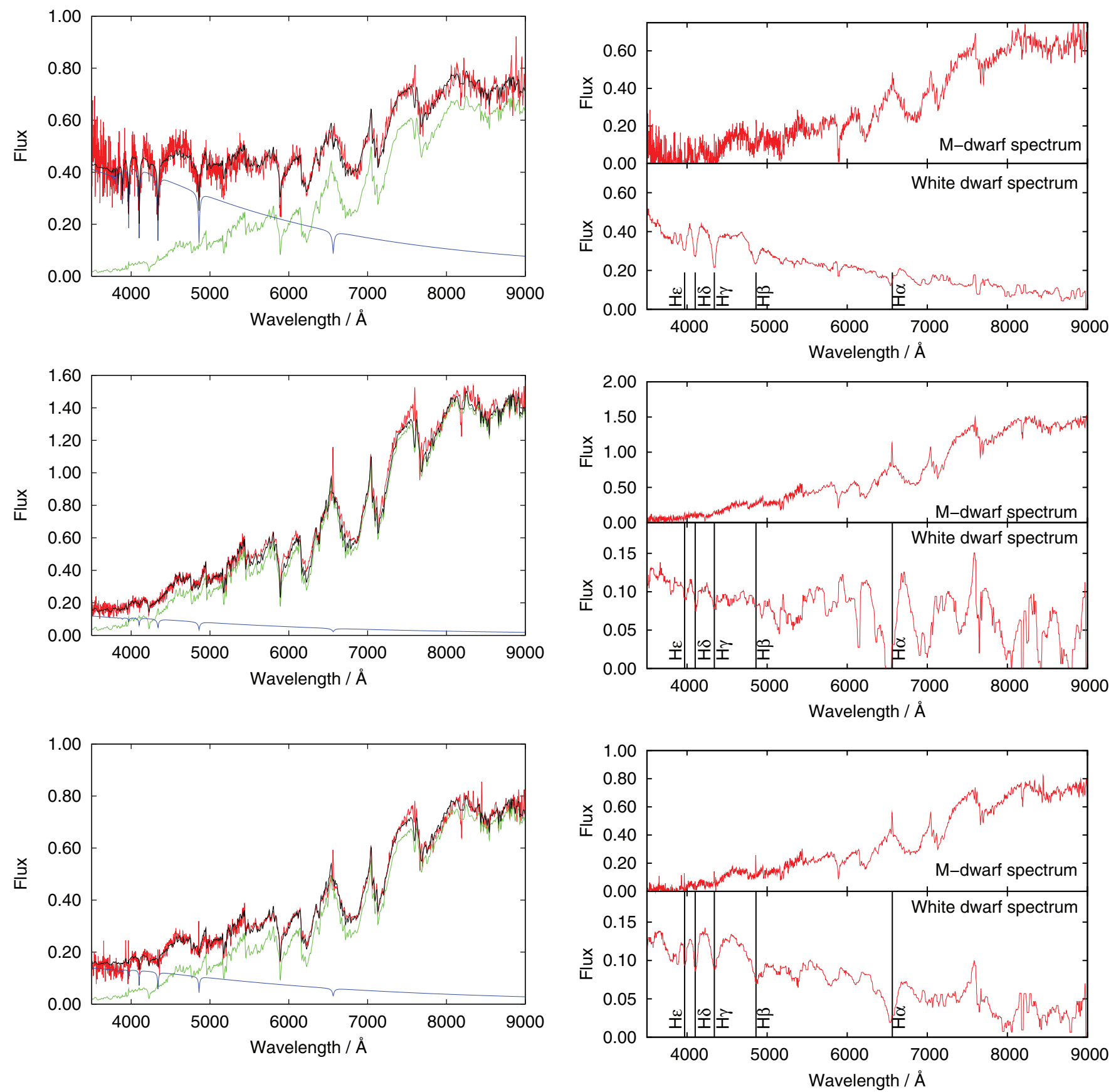

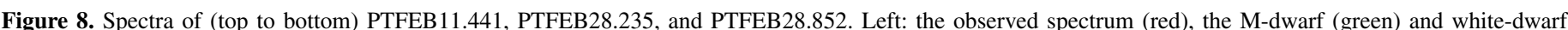

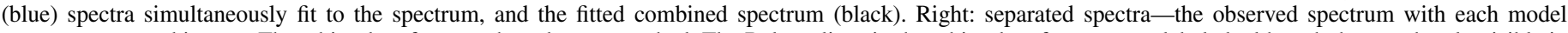

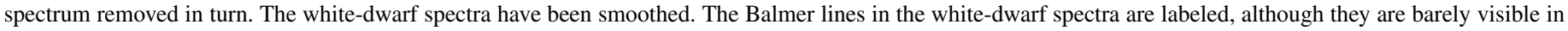
PTFEB28.235. We note that in each case the separated $\mathrm{H} \alpha$ absorption is offset in wavelength.

(A color version of this figure is available in the online journal.)

a $25 \%$ distance uncertainty (Kraus \& Hillenbrand 2007; Law et al. 2010a), and $\pm 1-2$ spectral subclass uncertainty (Kraus \& Hillenbrand 2007); the SED estimates for the spectral types are consistent with those derived from the two-component fits to our low-resolution spectroscopy. We also attempted to determine the M-dwarf spectral type from the TiO5 narrowband spectroscopic index (Gizis 1997), but found implausibly high values of the index, suggesting possible contamination by white-dwarf emission. In the following sections, we adopt the spectral types estimated from the two-component fits to the low-resolution spectra, as that method yields the uncertainty in the spectral type.

We estimate the M-dwarf masses from the spectral type vs. mass calibrations detailed by Delfosse et al. (2000), assuming they are on the main sequence and have solar metallicity and age. All three of our targets have a measured spectral type of M3, giving an estimated mass of $0.35 \pm 0.05 M_{\odot}$. We note that a small fraction of the systems measured by Delfosse et al. (2000) have much lower masses at the M3 spectral type, for reasons that are still unclear. To derive the masses in an alternative 

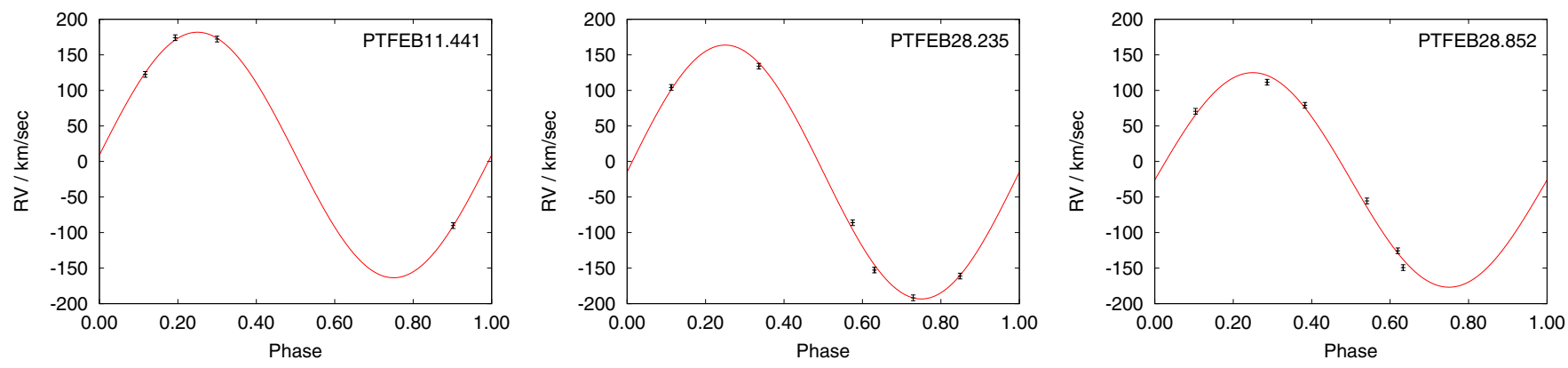

Figure 9. Radial-velocity orbit fits for each of the targets.

(A color version of this figure is available in the online journal.)

Table 4

Spectroscopic Orbit Properties

\begin{tabular}{llrc}
\hline \hline Name & $\begin{array}{c}K_{1} \\
\left(\mathrm{~km} \mathrm{~s}^{-1}\right)\end{array}$ & $\begin{array}{c}V_{0} \\
\left(\mathrm{~km} \mathrm{~s}^{-1}\right)\end{array}$ & $\begin{array}{c}\text { Mass Function } \\
(F)\end{array}$ \\
\hline PTFEB11.441 & $173 \pm 5$ & $9 \pm 4$ & $0.14 \pm 0.04$ \\
PTFEB28.235 & $179 \pm 7$ & $-15 \pm 5$ & $0.23 \pm 0.03$ \\
PTFEB28.852 & $151 \pm 10$ & $-26 \pm 7$ & $0.16 \pm 0.03$ \\
\hline
\end{tabular}

manner, we estimated the M-dwarf effective temperatures by the relation described in Luhman (1999). We then combined those estimates with the 5 Gyr isochrones of solar-metallicity stars in Baraffe et al. (1998) to estimate the stellar masses. These relations also predict a much lower mass for our M-dwarf targets, of $0.23_{0.04}^{+0.06} M_{\odot}$. In the following sections, we adopt the higher masses measured by Delfosse et al. (2000). We, however, note that two-component RV measurements for our systems giving masses for each component would help understand the mass vs. spectral type relation in this mass range.

We estimate the M-dwarfs' radii from a fit to the eclipsingbinary-derived mass/radius relation. We note that M-dwarfs in close binaries appear to be biased toward larger radii compared to systems with wider orbital radii (Kraus et al. 2011). We adopt a mass/radius relation for close ( $<1$ day period) binaries based on the measurements summarized by Kraus et al. (2011):

$$
R=1.096 M-0.052 \text { for } 0.1 M_{\odot}<M<0.7 M_{\odot},
$$

where $M$ is the mass in solar masses and $R$ is the radius in solar radii. The radii in this relation are approximately 5\%-10\% larger than both those measured for larger orbital radius systems and theoretical expectations (e.g., Baraffe et al. 1998; Kraus et al. 2011). The results are summarized in Table 3 .

\subsection{White-dwarf Properties}

We determine the white-dwarf masses from the measured RV curves, assuming the M-dwarf masses derived above. We fit a simple model to the velocity curves (Figure 9 and Table 4), assuming zero eccentricity because of the short circularization timescales for these compact systems (for example, see Devor et al. 2008). PTFEB28.235 and PTFEB28.852 have sufficient numbers of RV points to allow an eccentric orbit fit, but the fitted eccentricities are consistent with being zero. The whitedwarf masses $\left(M_{\mathrm{WD}}\right)$ are related to the fit mass function $(F)$, the M-dwarf masses $\left(M_{\mathrm{MD}}\right)$, and the inclination $(i)$ by

$$
F=\frac{M_{\mathrm{WD}}^{3} \sin ^{3} i}{\left(M_{\mathrm{MD}}+M_{\mathrm{WD}}\right)^{2}}
$$

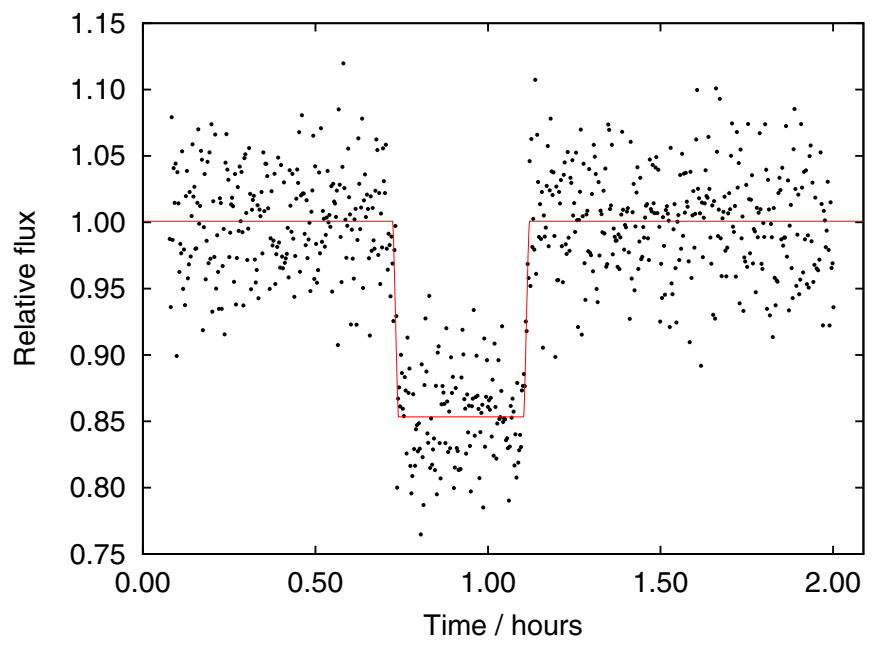

Figure 10. 10 s cadence observations of PTFEB11.441 from the BOS telescope (black points). A clear filter was used to increase the signal from the target. The best-fit eclipse model is shown in red.

(A color version of this figure is available in the online journal.)

which can be solved as a cubic equation. The results are summarized in Table 5. The inclination has only a weak effect on the derived white-dwarf masses; we marginalize it across all ranges allowed by the eclipse geometry.

We estimate the white-dwarf radii in two ways: first, models of the white-dwarf effective temperature, mass, and surface gravity cooling curves, and second a direct fit to the white dwarf's eclipse light curve.

Our model radius estimates are based on the Bergeron cooling curves for DA white dwarfs (Holberg \& Bergeron 2006; Kowalski \& Saumon 2006; Tremblay et al. 2011; Bergeron et al. 2011), for which we derive the allowed surface gravity range from the white-dwarf mass range measured above. The effective temperature has only a small effect on the surface gravity estimates and so we fix it at the best-fit value from the low-resolution spectra.

\subsection{Light-curve Models}

We obtain a direct upper limit for the white-dwarf radius in the PTFEB11.441 system from high-cadence eclipse data acquired with the BOS telescope (Figure 10). Using a clear filter to boost the signal levels, we were able to obtain $10 \mathrm{~s}$ cadence data with $\approx 5 \%$ photometric stability, sufficient time resolution to give stringent constraints on the ingress and egress timing of the system. 
Table 5

White-dwarf Properties

\begin{tabular}{lcccc}
\hline \hline Name & $M_{\mathrm{WD}}$ & $R_{\mathrm{WD}, \text { model }}$ & $R_{\mathrm{WD}, \text { spec. }}$ & $R_{\mathrm{WD}, \text { direct }}$ \\
\hline PTFEB11.441 & $0.54 \pm 0.05 M_{\odot}$ & $0.014 \pm 0.002 R_{\odot}$ & $0.010 R_{\odot}$ & $<0.025 R_{\odot}(95 \%)$ \\
PTFEB28.235 & $0.60 \pm 0.06 M_{\odot}$ & $0.013 \pm 0.002 R_{\odot}$ & $\ldots$ & $\ldots$ \\
PTFEB28.852 & $0.49 \pm 0.06 M_{\odot}$ & $0.015 \pm 0.002 R_{\odot}$ & $\ldots$ & $\ldots$ \\
\hline
\end{tabular}

Notes. Derived masses and radii for the white dwarfs in the eclipsing binary systems. $M_{\mathrm{WD}}$ is the stellar mass, $R_{\mathrm{WD} \text {,model }}$ is the radius derived from the mass and the Bergeron cooling models, $R_{\mathrm{WD}, \text { spec. }}$ is the radius derived from direct fits of the Koester (2010) model spectra Balmer lines to the white-dwarf spectrum, and $R_{\mathrm{WD} \text {, direct }}$ is the $95 \%$ confidence upper limit measured from the eclipse ingress and egress shapes.

We fit the light curves with a simple two-spherical-body model. The flat-bottomed light curves show that the white dwarf is completely eclipsed, allowing a simple model based on the Mandel \& Agol (2002) transiting-planet model to be used. We modified the model to allow both stars to be moving relative to a common center of mass, to allow both bodies to be selfluminous, and to include a linear limb-darkening term for the white dwarf.

Inspection of the light curves shows that the white-dwarf ingress and egress is at best marginally resolved, and so we only attempt to estimate an upper limit for the white-dwarf radius. We obtain a maximal estimate of the white-dwarf radius when the inclination is assumed to be $90^{\circ}$, and so we fix the inclination to that value in our modeling. For the white dwarf, we conservatively use a linear limb-darkening coefficient of 0.5 ; we tested a range of coefficients and found that the limb darkening has no significant effect on the radius estimates, because the eclipse ingress and egress are barely resolved. We fix the M-dwarf radius and the stellar mass ratio $q$ during the fits, although we marginalize over the estimated $1 \sigma$ ranges of both parameters.

We vary the semimajor axis of the system, the white-dwarf radius, and the flux from both components (and hence the contribution from the system's unresolved wide companion). When the inclination is fixed at $90^{\circ}$, the best-fit semimajor axis value is within the error bars of our measurements based on the eclipse timing and the estimated $\mathrm{M}$-dwarf radius, although those estimates suggest that a somewhat lower inclination may be preferable (which would lead to a smaller white-dwarf radius). We estimate the range of fitted parameters and a 95\% upper limit on the white-dwarf radius using the Bootstrap algorithm.

This procedure puts the upper-limit white-dwarf radius in the PTFEB 11.441 system as $<0.025 R_{\odot}$ at $95 \%$ confidence, a value consistent with the radii estimated from cooling models and log $g$ fits to the white-dwarf's Balmer lines. The fitted semimajor axes are around $10 \%$ larger than those expected from the period of the system and the estimated masses of the components, suggesting we have slightly overestimated the mass of at least one component, most likely the M-dwarf in the system.

\subsection{M-dwarf Activity}

All three systems show evidence of active M-dwarfs, including strong $\mathrm{H} \alpha$ emission and variability on a variety of timescales. None are detected in the ROSAT All-Sky Faint Source Catalog (Voges et al. 2000), although the M-dwarfs would have to be exceptionally active to be detected at these distances (e.g., Law et al. 2008).

The positioning of PTFEB11.441 near M31 allows more archival data to be found. The system is co-incident with

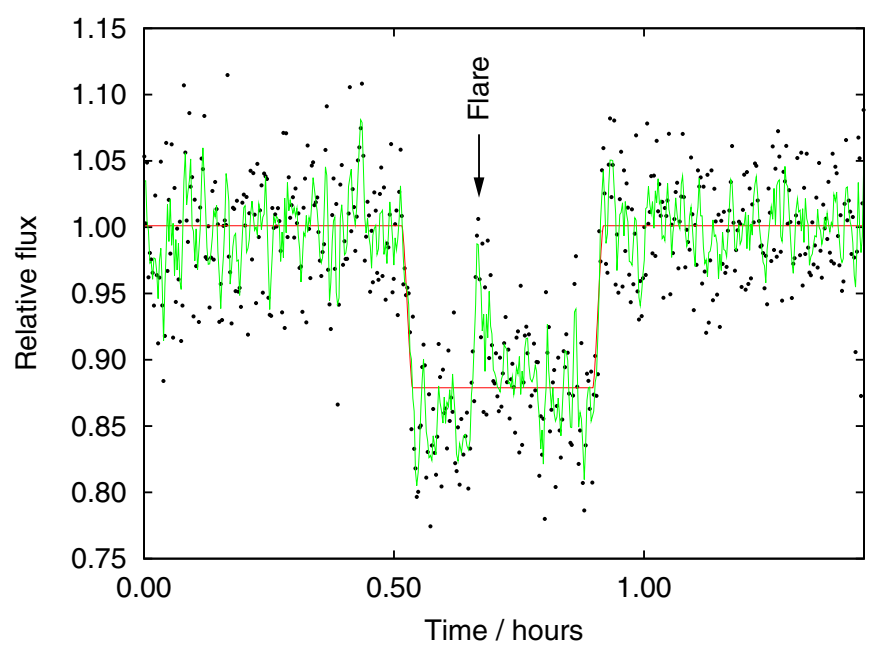

Figure 11. Flare event during a high-cadence observation of PTFEB11.441. The green line shows a $30 \mathrm{~s}$ moving average of the $10 \mathrm{~s}$ cadence photometry (black points). The red line shows the best-fit eclipse light curve.

(A color version of this figure is available in the online journal.)

the Chandra X-ray source ${ }^{17}$ CXO J004545.8+415029 $(2.8 \times$ $10^{-13} \mathrm{erg} \mathrm{s}^{-1} \mathrm{~cm}^{-2}$ ) located within $1 \sigma$ of the PTF position for the system. Using the 2MASS $K$-band photometry of the system (which is dominated by the M-dwarf luminosity) and the empirical relations in Leggett (1992) and Tinney et al. (1993) to estimate the bolometric luminosity of the system, we calculate $\log \left(L_{x} / L_{\text {bol }}\right)=-1.5$. Although the flare event described below suggests that the M-dwarf in the system is indeed active, this is an extremely high activity compared to typical field M-dwarfs (e.g., Delfosse et al. 1998; Law et al. 2008). This suggests that the X-ray detection is a spurious association with the system, the X-ray observation was taken in a time of extreme activity, or that the bulk of the X-ray emission does not originate in the M-dwarf chromosphere.

We also identified a flare event during one high-cadence observation of PTFEB11.441 (Figure 11). The observation was taken without a filter to boost our $\mathrm{S} / \mathrm{N}$, limiting the detailed interpretation of the event. The shape of the event is as expected for an M-dwarf flare, with a rapid rise followed by an approximately exponential decay.

\subsection{Out-of-eclipse Variations}

The light curves show out-of-eclipse sinusoidal variability correlated with the orbit. The overall shape is consistent with irradiation of the side of the M-dwarf facing the white dwarf, where the flux maximum is expected half an orbital phase

\footnotetext{
17 From the Chandra Source Catalog (Evans et al. 2010).
} 


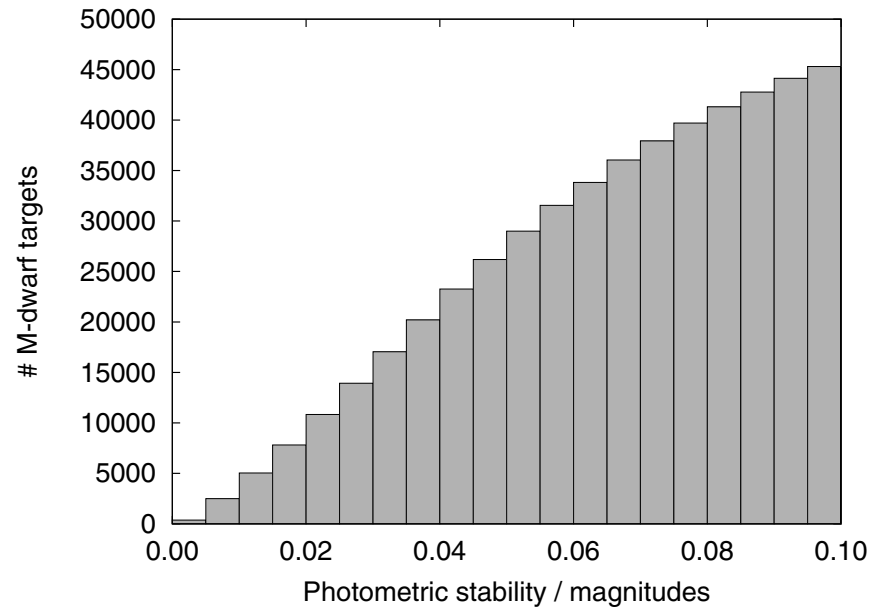

Figure 12. Cumulative number of M-dwarf targets searched by the PTF/M-dwarfs survey in the first year of operations, as a function of achieved photometric stability. M-dwarfs in the PTF/M-dwarf fields are selected and confirmed on the basis of 2MASS, USNO-B1, and (where available) SDSS colors, along with proper motions determined from 2MASS and USNO-B1 positions. We require a high-confidence photometric color fit, along with a $2 \sigma$ proper-motion detection to rule out giant interlopers.

from the occultation. Other mechanisms that may affect the out-of-eclipse light curves of such compact systems are tidal ellipsoidal distortion and the beaming effect (e.g., Zucker et al. 2007; Shporer et al. 2010a), but simple estimates show that both effects are expected to be at the $1 \%$ level or below for the binaries presented here and so cannot be identified in the available data.

PTFEB11.441 shows much lower levels of out-of-eclipse variations than the other two systems. This is most likely due to light from its possible companion diluting the variations (and eclipse depths) in unresolved photometry of the system.

\section{DISCUSSION AND CONCLUSIONS}

The discovery of only three white-dwarf/M-dwarf binaries in the PTF/M-dwarfs survey suggests a low incidence for such systems. This phase of the survey has covered $\approx 45,000$ M-dwarfs with sufficient precision to detect short-period systems with eclipse depths similar to those shown here (Figure 12). The survey's detection efficiency for systems with fractionalday periods is near $100 \%$ (Figure 13). The geometric probability of eclipse is $5 \%-15 \%$ for an early-M-dwarf primary in a roughly equal-mass half-day-period binary with a white dwarf. Taken together, the detection of three systems implies that $0.08 \%+0.05 \%(90 \%$ confidence) of M-dwarfs are in shortperiod post-common-envelope white-dwarf/M-dwarf binaries where the optical light is dominated by the M-dwarf.

The periods of the detected systems are longer than most of the eclipsing M-dwarf/white-dwarf systems found in Drake et al. (2010; Figure 14), which offers a similar comparison sample derived from a survey targeted at deep eclipses of white-dwarf systems. Our survey is designed to detect longerperiod transiting exoplanets, so this is not in itself surprising. However, our lack of detections at shorter periods, despite our nearly $100 \%$ detection efficiency for such systems, suggests that binaries including these relatively low-temperature white dwarfs preferentially occur at relatively large orbital radii.

Our survey is sensitive to systems with periods several times those noted here, and to M-dwarf/white-dwarf systems containing much earlier M-dwarfs than detected here. As discussed in Davis et al. (2010), Rebassa-Mansergas et al. (2008), and Nebot

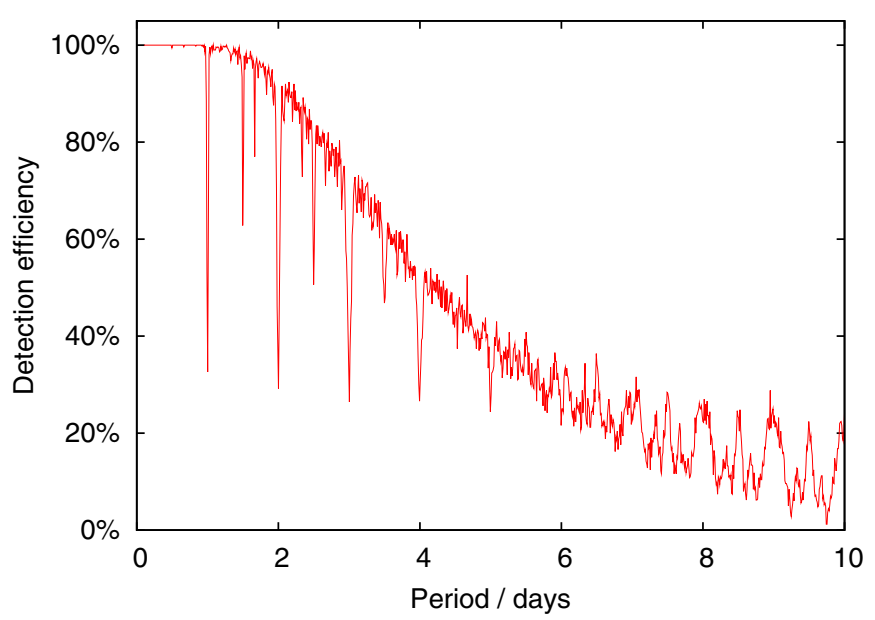

Figure 13. Eclipse-detection efficiency for a typical PTF/M-dwarfs observing scenario, based on simulations including the actual observing cadences of the survey. The detection efficiency is the probability that at least two eclipses will be observed from an eclipsing target with a specified period, after observing window and weather effects have been taken into account. The low efficiencies around integer numbers of days at short periods are due to the likelihood of an eclipse always occurring in the daytime; the same effect allows some long-period binaries to be picked up if their eclipses always occur at night.

(A color version of this figure is available in the online journal.)

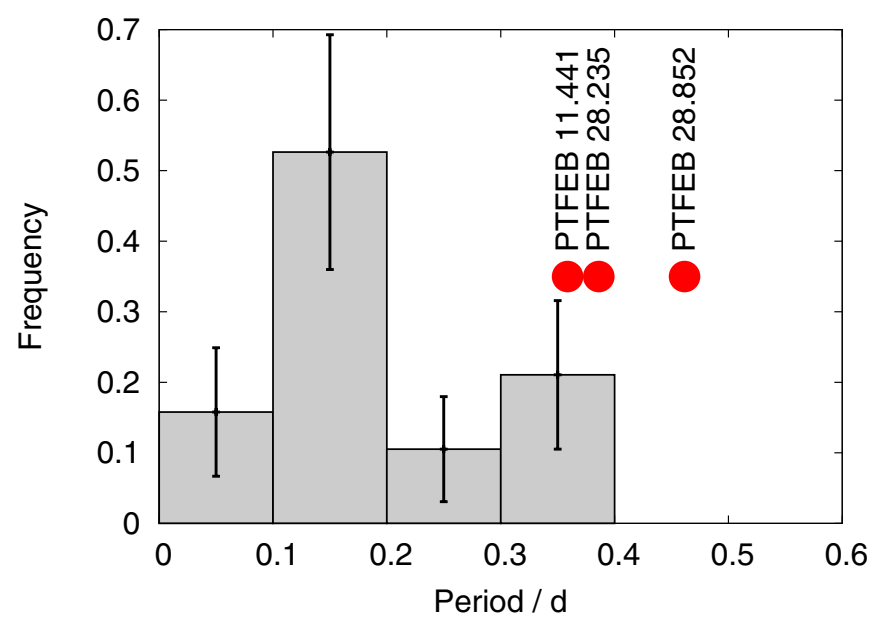

Figure 14. Periods of our three detected systems compared to the distribution of periods of the white-dwarf-dominated eclipsing systems found by Drake et al. (2010), which were all found in a single survey and so offer a useful comparison sample.

(A color version of this figure is available in the online journal.)

Gómez-Morán et al. (2011), the lack of longer-period systems may be an intrinsic feature of the population. The lack of earlier companions in our survey is likely to provide further constraints on common-envelope evolutionary models (Davis et al. 2010).

Using the analysis described in Schreiber \& Gänsicke (2003), we can explore the future evolution of these systems. Assuming angular momentum loss due to gravitational radiation alone, we estimate that mass transfer will begin after approximately $10^{10}$ years at orbital periods of 0.08 days (PTFEB28.235) and 0.09 days (PTFEB11.441 and PTFEB28.852). All the systems are expected to start transfer within the cataclysmic variable period gap, reinforcing the relative lack of long-period $\mathrm{CV}$ progenitors pointed out by Schreiber \& Gänsicke (2003).

These eclipsing binaries appear as M-dwarfs when selected by optical and infrared colors, and they display transit-like light curves which can have arbitrarily small depths. They appear 
to be at least three times more common than transiting giant planets in the PTF/M-dwarfs survey. These properties make these systems an important false-positive for current and future transiting planet searches around M-dwarfs. Fortunately, they can be distinguished from true transiting planets with small amounts of extra data, using the methods detailed below. The methods are ordered by increasing difficulty, starting with those which require only the discovery light curves.

1. Eclipse duration. The increased mass of the system compared to a M-dwarf/planet system leads to a shorter eclipse; high-S/N and high-cadence light curves are, however, required to distinguish this case from a lower-inclination system.

2. Eclipse shape. The eclipse of the white dwarf by the M-dwarf provides an exactly flat-bottomed eclipse outside the short ingress and egress periods. With high-precision photometry this can be distinguished from a true transiting planet which will show the effects of limb darkening as it passes in front of the M-dwarf.

3. Out-of-eclipse variability. All of our systems show out-ofeclipse variability at levels which are orders of magnitude greater than that expected for planetary systems (see Drake 2003 for details of a similar selection method for planetary transits around solar-type stars).

4. UV emission. Two of our new systems showed UV emission and the third has possible UV emission. Where data are available, the presence of UV (or even $u$ band) emission suggests the existence of something other than an M-dwarf in the system.

5. Low-resolution spectroscopy. Like the three targets presented in this paper, a low-resolution spectrum could be decomposed into white-dwarf and M-dwarf components. Faint, low-temperature white dwarfs may, however, be hard to detect with this technique.

6. Multi-color photometry. These systems show a strong variation in eclipse depth with wavelength (depending on the relative temperatures of the white dwarf and M-dwarf and their ratio of radii).

7. Radial velocities. These systems have RV amplitudes at least $500 \times$ larger than expected for a planet with the same period. Just two few $\mathrm{km} / \mathrm{sec}$ precision RV data points are sufficient to discriminate these systems from transiting planet systems.

Of these methods, multi-color photometry through eclipse is probably the most time-efficient method of detecting systems like this. As large-scale transiting planet surveys of M-dwarfs start up and continue, many more systems in this interesting parameter space are likely to be found.

Follow-up precision photometry and RVs will allow direct measurements of the masses and radii of all components of these systems. As the white dwarf transits across the disk of the M-dwarf the transit depth is expected to be around $1 \mathrm{mmag}$, although lensing by the white dwarf (e.g., Marsh 2001) will make the transit shallower than expected from only geometric considerations (e.g., Steinfadt et al. 2010). High-cadence and high-precision photometry of the whitedwarf eclipse (occultation) ingress and egress may be the best approach to directly measure the white-dwarf radii (the M-dwarf flare frequency is low enough (Kowalski et al. 2009) that the presence of M-dwarf variability such as that displayed in Figure 11 will not preclude this measurement). Furthermore, small asymmetries in the ingress and egress light curve due to the photometric Rossiter-McLaughlin effect (Shporer et al. 2012; Groot 2012) can allow a measurement of the white-dwarf spin-orbit alignment and rotation velocity. With these methods, this new group of systems will fill a poorly covered range of the white-dwarf and M-dwarf mass/radius relations.

We thank Michael Kandrashoff, Jieun Choi, and Peter Blanchard for observations at Lick Observatory, and thank the anonymous referee for their constructive suggestions and swift report. Observations were obtained with the Samuel Oschin Telescope and the 60 inch telescope at the Palomar Observatory as part of the Palomar Transient Factory project, a scientific collaboration between the California Institute of Technology, Columbia University, Las Cumbres Observatory, the Lawrence Berkeley National Laboratory, the National Energy Research Scientific Computing Center, the University of Oxford, and the Weizmann Institute of Science. Some of the data presented herein were obtained at the W. M. Keck Observatory, which is operated as a scientific partnership among the California Institute of Technology, the University of California, and NASA; the observatory was made possible by the generous financial support of the W. M. Keck Foundation. We recognize and acknowledge the very significant cultural role and reverence that the summit of Mauna Kea has always had within the indigenous Hawaiian community; we are most fortunate to have the opportunity to conduct observations from this mountain. The Byrne Observatory at Sedgwick (BOS) is operated by the Las Cumbres Observatory Global Telescope Network and is located at the Sedgwick Reserve, a part of the University of California Natural Reserve System. The RoboAO system is supported by collaborating partner institutions, the California Institute of Technology, and the Inter-University Centre for Astronomy and Astrophysics, and by the National Science Foundation under Grant Nos. AST-0906060 and AST0960343. This paper uses observations obtained with the FTN observatory of the Las Cumbres Observatory Global Telescope. This research has also made use of the SIMBAD database, operated at CDS, Strasbourg, France. The radial velocity analysis in this paper used two existing data analysis pipelines, MAKEE by Tom Barlow and BFall by Slavek Rucinski; we gratefully acknowledge their contribution to the field in developing and supporting this software. N.M.L. is supported by a Dunlap Fellowship at University of Toronto. A.L.K. was supported by NASA through Hubble Fellowship Grant 51257.01 awarded by STScI, which is operated by AURA, Inc., for ANSA, under contract NAS 5-26555. M.M.K. acknowledges support from the Hubble Fellowship and Carnegie-Princeton Fellowship. A.V.F. and his group at UC Berkeley acknowledge generous financial assistance from Gary and Cynthia Bengier, the Richard \& Rhoda Goldman Fund, the TABASGO Foundation, and NSF Grant AST-0908886.

Facilities: PO:1.2m, Keck:I, LCOGT

\section{REFERENCES}

Agüeros, M. A., Covey, K. R., Lemonias, J. J., et al. 2011, ApJ, 740, 110 Baraffe, I., Chabrier, G., Allard, F., \& Hauschildt, P. H. 1998, A\&A, 337, 403 Baranec, C., Riddle, R., Ramaprakash, A., et al. 2011, in Adaptive Optics: Methods, Analysis and Applications (Washington, DC: Optical Society of America), AWA2

Bergeron, P., Wesemael, F., Dufour, P., et al. 2011, ApJ, 737, 28

Bertin, E., \& Arnouts, S. 1996, A\&AS, 117, 393

Bianchi, L., Rodriguez-Merino, L., Viton, M., et al. 2007, ApJS, 173, 659

Borucki, W. J., Koch, D. G., Basri, G., et al. 2011, ApJ, 736, 19

Bruch, A., \& Diaz, M. P. 1998, AJ, 116, 908

Charbonneau, D., Berta, Z. K., Irwin, J., et al. 2009, Nature, 462, 891 
Davis, P. J., Kolb, U., \& Willems, B. 2010, MNRAS, 403, 179

Delfosse, X., Forveille, T., Perrier, C., \& Mayor, M. 1998, A\&A, 331, 581

Delfosse, X., Forveille, T., Ségransan, D., et al. 2000, A\&A, 364, 217

Devor, J., Charbonneau, D., O’Donovan, F. T., Mandushev, G., \& Torres, G. 2008, AJ, 135, 850

Dhital, S., West, A. A., Stassun, K. G., \& Bochanski, J. J. 2010, AJ, 139,2566

Drake, A. J. 2003, ApJ, 589, 1020

Drake, A. J., Beshore, E., Catelan, M., et al. 2010, arXiv:1009.3048

Eastman, J., Siverd, R., \& Gaudi, B. S. 2010, PASP, 122, 935

Evans, I. N., Primini, F. A., Glotfelty, K. J., et al. 2010, ApJS, 189, 37

Filippenko, A. V. 1982, PASP, 94, 715

Foley, R. J., Papenkova, M. S., Swift, B. J., et al. 2003, PASP, 115, 1220

Fulbright, M. S., Liebert, J., Bergeron, P., \& Green, R. 1993, ApJ, 406, 240

Gizis, J. E. 1997, AJ, 113, 806

Green, R. F., Richstone, D. O., \& Schmidt, M. 1978, ApJ, 224, 892

Groot, P. J. 2012, ApJ, 745, 55

Holberg, J. B., \& Bergeron, P. 2006, AJ, 132, 1221

Horne, K. 1986, PASP, 98, 609

Irwin, J., Buchhave, L., Berta, Z. K., et al. 2010, ApJ, 718, 1353

Klöckner, A., Pinto, N., Lee, Y., et al. 2009, arXiv:0911.3456K

Koester, D. 2010, Mem. Soc. Astron. Ital., 81, 921

Kovács, G., Zucker, S., \& Mazeh, T. 2002, A\&A, 391, 369

Kowalski, A. F., Hawley, S. L., Hilton, E. J., et al. 2009, AJ, 138, 633

Kowalski, P. M., \& Saumon, D. 2006, ApJ, 651, L137

Kraus, A. L., \& Hillenbrand, L. A. 2007, AJ, 134, 2340

Kraus, A. L., Tucker, R. A., Thompson, M. I., Craine, E. R., \& Hillenbrand, L. A. 2011, ApJ, 728, 48

Law, N. M. 2007, Observatory, 127, 71

Law, N. M., Dekany, R. G., Rahmer, G., et al. 2010a, Proc. SPIE, 7735, $122 \mathrm{~L}$

Law, N. M., Dhital, S., Kraus, A., Stassun, K. G., \& West, A. A. 2010b, ApJ, 720,1727

Law, N. M., Hodgkin, S. T., \& Mackay, C. D. 2008, MNRAS, 384, 150

Law, N. M., Kraus, A. L., Street, R. R., et al. 2011, in ASP Conf. Ser. 448, Cool Stars, Stellar Systems, and the Sun, ed. C. Johns-Krull, M. K. Browning, \& A. A. West (San Francisco, CA: ASP), 1367

Law, N. M., Kulkarni, S. R., Dekany, R. G., et al. 2009a, PASP, 121, 1395

Law, N. M., Mackay, C. D., Dekany, R. G., et al. 2009b, ApJ, 692, 924

Leggett, S. K. 1992, ApJS, 82, 351

Luhman, K. L. 1999, ApJ, 525, 466

Mandel, K., \& Agol, E. 2002, ApJ, 580, L171

Marsh, T. R. 2001, MNRAS, 324, 547

Matheson, T., Filippenko, A. V., Ho, L. C., Barth, A. J., \& Leonard, D. C. 2000, AJ, 120, 1499

Miller, J. S., \& Stone, R. P. S. 1993, Lick Obs. Technical Report 66, Lick Obs., Santa Cruz

Monet, D. G., Levine, S. E., Canzian, B., et al. 2003, AJ, 125, 984
Nebot Gómez-Morán, A., Gänsicke, B. T., Schreiber, M. R., et al. 2011, A\&A, 536, A43

Nebot Gómez-Morán, A., Schwope, A. D., Schreiber, M. R., et al. 2009, A\&A, 495, 561

Nelson, B., \& Young, A. 1970, PASP, 82, 699

Nidever, D. L., Marcy, G. W., Butler, R. P., Fischer, D. A., \& Vogt, S. S. 2002, ApJS, 141, 503

Parsons, S. G., Marsh, T. R., Gänsicke, B. T., Drake, A. J., \& Koester, D. 2011, ApJ, 735, L30

Parsons, S. G., Marsh, T. R., Gänsicke, B. T., et al. 2012, MNRAS, 420, 3281

Pickles, A. J. 1998, PASP, 110, 863

Press, W. H., Teukolsky, S. A., Vetterling, W. T., \& Flannery, B. P. 1992, in Numerical Recipes in C. The Art of Scientific Computing, ed. W. H. Press, S. A. Teukolsky, W. T. Vetterling, \& B. P. Flannery (Cambridge: Cambridge University Press), 691

Pyrzas, S., Gaensicke, B. T., Brady, S., et al. 2012, MNRAS, 419, 814

Pyrzas, S., Gänsicke, B. T., Marsh, T. R., et al. 2009, MNRAS, 394, 978

Rahmer, G., Smith, R., Velur, V., et al. 2008, Proc. SPIE, 7014, 163R

Rau, A., Kulkarni, S. R., Law, N. M., et al. 2009, PASP, 121, 1334

Rebassa-Mansergas, A., Gänsicke, B. T., Schreiber, M. R., et al. 2008, MNRAS, 390, 1635

Rebassa-Mansergas, A., Nebot Gomez-Moran, A., Schreiber, M. R., et al. 2012, MNRAS, 419, 806

Rucinski, S. 1999, in ASP Conf. Ser. 185, IAU Colloq. 170: Precise Stellar Radial Velocities, ed. J. B. Hearnshaw \& C. D. Scarfe (San Francisco, CA: ASP), 82

Schlegel, D. J., Finkbeiner, D. P., \& Davis, M. 1998, ApJ, 500, 525

Schreiber, M. R., \& Gänsicke, B. T. 2003, A\&A, 406, 305

Shporer, A., Brown, T., Mazeh, T., \& Zucker, S. 2012, New Astron., 17, 309

Shporer, A., Kaplan, D. L., Steinfadt, J. D. R., et al. 2010a, ApJ, 725, L200

Shporer, A., Winn, J. N., Dreizler, S., et al. 2010b, ApJ, 722, 880

Sipőcz, B., Kovács, G., Pinfield, D., \& Hodgkin, S. 2011, in Detection and Dynamics of Transiting Exoplanets, St. Michel l'Observatoire, France, ed. F. Bouchy, R. Díaz, \& C. Moutou, EPJ Web of Conferences, Vol. 11 (Berlin: Springer), 06003

Skrutskie, M. F., Cutri, R. M., Stiening, R., et al. 2006, AJ, 131, 1163

Smolčić, V., Ivezić, Ž., Knapp, G. R., et al. 2004, ApJ, 615, L141

Steinfadt, J. D. R., Kaplan, D. L., Shporer, A., Bildsten, L., \& Howell, S. B. 2010, ApJ, 716, L146

Tingley, B. 2003, A\&A, 408, L5

Tinney, C. G., Mould, J. R., \& Reid, I. N. 1993, AJ, 105, 1045

Tremblay, P.-E., \& Bergeron, P. 2009, ApJ, 696, 1755

Tremblay, P.-E., Bergeron, P., \& Gianninas, A. 2011, ApJ, 730, 128

Voges, W., Aschenbach, B., Boller, T., et al. 2000, IAU Circ., 7432, 3

Vogt, S. S., Allen, S. L., Bigelow, B. C., et al. 1994, Proc. SPIE, 2198, 362

Wade, R. A., \& Horne, K. 1988, ApJ, 324, 411

York, D. G., Adelman, J., Anderson, J. E., Jr., et al. 2000, AJ, 120, 1579

Zucker, S., Mazeh, T., \& Alexander, T. 2007, ApJ, 670, 1326 